Perspective

\title{
An Evidence-Based Multidisciplinary Approach Focused on Creating Algorithms for Targeted Therapy of Infection-Related Ventilator-Associated Complications (IVACs) Caused by Pseudomonas aeruginosa and Acinetobacter baumannii in Critically Ill Adult Patients
}

\author{
Milo Gatti ${ }^{1,2}$ (D), Bruno Viaggi ${ }^{3}$, Gian Maria Rossolini ${ }^{4,5,6}$, Federico Pea ${ }^{1,2, * \mathbb{D}}$ and Pierluigi Viale ${ }^{1,7}$ \\ check for \\ updates \\ Citation: Gatti, M.; Viaggi, B.; \\ Rossolini, G.M.; Pea, F.; Viale, P. An \\ 1 Department of Medical and Surgical Sciences, Alma Mater Studiorum University of Bologna, \\ 40138 Bologna, Italy; milo.gatti2@unibo.it (M.G.); pierluigi.viale@unibo.it (P.V.) \\ 2 SSD Clinical Pharmacology, Department for Integrated Infectious Risk Management, IRCCS Azienda \\ Ospedaliero-Universitaria di Bologna, 40138 Bologna, Italy \\ 3 Neurointensive Care Unit, Department of Anesthesiology, Careggi University Hospital, 50134 Florence, Italy; \\ bruno.viaggi@gmail.com \\ 4 Department of Experimental and Clinical Medicine, University of Florence, 50134 Florence, Italy; \\ gianmaria.rossolini@unifi.it \\ 5 Microbiology and Virology Unit, Florence Careggi University Hospital, 50134 Florence, Italy \\ 6 IRCCS Fondazione Don Carlo Gnocchi, 50143 Florence, Italy \\ 7 Infectious Diseases Unit, Department for Integrated Infectious Risk Management, IRCCS Azienda \\ Ospedaliero-Universitaria di Bologna, 40126 Bologna, Italy \\ * Correspondence: federico.pea@unibo.it
} Evidence-Based Multidisciplinary Approach Focused on Creating Algorithms for Targeted Therapy of Infection-Related VentilatorAssociated Complications (IVACs) Caused by Pseudomonas aeruginosa and Acinetobacter baumannii in Critically Ill Adult Patients. Antibiotics 2022, 11, 33. https:// doi.org/10.3390/antibiotics11010033 Academic Editors: Joerg Overhage and Giovanni Di Bonaventura

Received: 31 October 2021

Accepted: 23 December 2021

Published: 28 December 2021

Publisher's Note: MDPI stays neutral with regard to jurisdictional claims in published maps and institutional affiliations.

Copyright: (C) 2021 by the authors. Licensee MDPI, Basel, Switzerland. This article is an open access article distributed under the terms and conditions of the Creative Commons Attribution (CC BY) license (https:// creativecommons.org/licenses/by/ $4.0 /)$.
Abstract: (1) Background: To develop evidence-based algorithms for targeted antibiotic therapy of infection-related ventilator-associated complications (IVACs) caused by non-fermenting Gramnegative pathogens. (2) Methods: A multidisciplinary team of four experts had several rounds of assessments for developing algorithms devoted to targeted antimicrobial therapy of IVACs caused by two non-fermenting Gram-negative pathogens. A literature search was performed on PubMedMEDLINE (until September 2021) to provide evidence for supporting therapeutic choices. Quality and strength of evidence was established according to a hierarchical scale of the study design. Six different algorithms with associated recommendations in terms of therapeutic choice and dosing optimization were suggested according to the susceptibility pattern of two non-fermenting Gram-negative pathogens: multi-susceptible Pseudomonas aeruginosa (PA), multidrug-resistant (MDR) metallobeta-lactamase (MBL)-negative-PA, MBL-positive-PA, carbapenem-susceptible Acinetobacter baumannii (AB), and carbapenem-resistant AB. (3) Results: Piperacillin-tazobactam or fourth-generation cephalosporins represent the first therapeutic choice in IVACs caused by multi-susceptible PA. A carbapenem-sparing approach favouring the administration of novel beta-lactam/beta-lactamase inhibitors should be pursued in the management of MDR-MBL-negative PA infections. Cefiderocol should be used as first-line therapy for the management of IVACs caused by MBL-producing-PA or carbapenem-resistant AB. Fosfomycin-based combination therapy, as well as inhaled colistin, could be considered as a reasonable alternative for the management of IVACs due to MDR-PA and carbapenem-resistant AB. (4) Conclusions: The implementation of algorithms focused on prompt revision of antibiotic regimens guided by results of conventional and rapid diagnostic methodologies, appropriate place in therapy of novel beta-lactams, implementation of strategies for sparing the broadest-spectrum antibiotics, and pharmacokinetic/pharmacodynamic optimization of antibiotic dosing regimens is strongly suggested.

Keywords: antimicrobial stewardship; infection-related ventilator-associated complications; nonfermenting Gram-negative pathogens; multidisciplinary taskforce; PK/PD dosing optimization; targeted antibiotic therapy 


\section{Introduction}

Infection-related ventilator-associated complications (IVACs) represent the most prevalent infective events in patients admitted to the intensive care unit (ICU) and requiring mechanical ventilation [1], accounting approximately for one-third of hospital-acquired pneumonia (HAP) cases [2]. IVACs are associated with high mortality rate (over 50\%) and with a remarkable impact on length of ICU stay, antibiotic use, and overall health care costs [2-5]. Gram-negative pathogens are responsible for the majority of HAP and ventilator-associated pneumonia (VAP), and among these, the non-fermenting Gram-negative pathogens (especially Pseudomonas aeruginosa and Acinetobacter baumannii) are responsible for a remarkable amount of IVACs in critically ill patients, second only to Staphylococcus aureus in terms of prevalence $[6,7]$.

Pseudomonas aeruginosa and Acinetobacter baumannii are both characterized by innate resistance mechanisms against multiple antimicrobials. Furthermore, these pathogens may easily acquire new resistances by different mobile elements, thus making extremely challenging the choice of appropriate antibiotic therapy in this setting [6-9]. Prompt initiation of empirical broad-spectrum antibiotic treatment is necessary in critically ill patients with suspected IVAC, including agents with activity against non-fermenting Gram-negative pathogens according to the existence of specific risk factors or in case of epidemiological settings characterized by high prevalence. In the last report on antimicrobial surveillance issued by the European Center for Disease Prevention and Control (ECDC) [10], the proportion of invasive isolates of multidrug resistant (MDR) Pseudomonas aeruginosa and carbapenem-resistant Acinetobacter baumannii accounted for $12.1 \%$ and $32.6 \%$, respectively. In the Italian context, the proportion of the invasive strains of MDR Pseudomonas aeruginosa is in line with European data (13.1\%), while that of carbapenem-resistant Acinetobacter baumannii isolates accounts for as much as 80\% [10]. However, once that the causative pathogen has been identified and its susceptibility pattern has been defined, therapy should be revised and targeted, as recommended by the Surviving Sepsis Campaign guidelines [11].

Microbiological confirmation of IVAC represents a crucial step for enabling targeted antibiotic therapy in critically ill patients. Unfortunately, traditional culture-based methods are time-consuming as they often require at least 1-2 days for pathogen isolation and an additional day for determination of the antibiotic susceptibility pattern. Furthermore, culture-based methods exhibit low sensitivity, as they may provide pathogen identification in less than $40 \%$ of patients with clinically diagnosed IVACs [12]. This has traditionally made challenging and often difficult a rapid implementation of targeted antibiotic therapy [10]. In recent years, the development of rapid molecular tests has revolutionized microbiological diagnosis in the pneumonia setting. These tests, based on syndromic panels, may provide fast identification of the respiratory pathogens coupled with detection of relevant genotypic markers of resistance. This approach is expected to have a huge impact in daily clinical practice, by making more and more applicable both targeted antibiotic therapy and antimicrobial stewardship strategies [12,13].

Appropriate targeted antimicrobial therapy, coupled with antibiotic dose optimization and implementation of antimicrobial stewardship programs, could simultaneously maximize efficacy, reduce antibiotic overconsumption, and minimize the development of resistance in ICU patients [14]. This could be achieved by means of the coordinated approach of a multidisciplinary task force, composed by the intensive care physician, the infectious disease consultant, the clinical microbiologist, and the MD or PharmD clinical pharmacologist. Early diagnosis with prompt identification of the causative pathogen of IVACs coupled with targeted antibiotic therapy based on antimicrobial susceptibility testing and on dosing optimization according to the pharmacokinetic/pharmacodynamic (PK/PD) concepts and the therapeutic drug monitoring (TDM)-guided approach may be fundamental for this purpose.

This multidisciplinary opinion article aims to develop evidence-based algorithms for targeted antibiotic therapy of IVACs caused by two non-fermenting Gram-negative 
pathogens in critically ill adult patients. The objective is to provide a useful guidance for intensive care physicians either in appropriately placing novel antimicrobial agents in lack of definitive evidence or in considering antimicrobial stewardship strategies for possibly sparing the broadest-spectrum antibiotics.

\section{Results}

2.1. Targeted Treatment of IVACs Caused by Pseudomonas aeruginosa in Critically Ill Adult Patients

Therapeutic algorithm for targeted treatment of IVACs caused by Pseudomonas aeruginosa in adult ICU patients is shown in Figure 1.
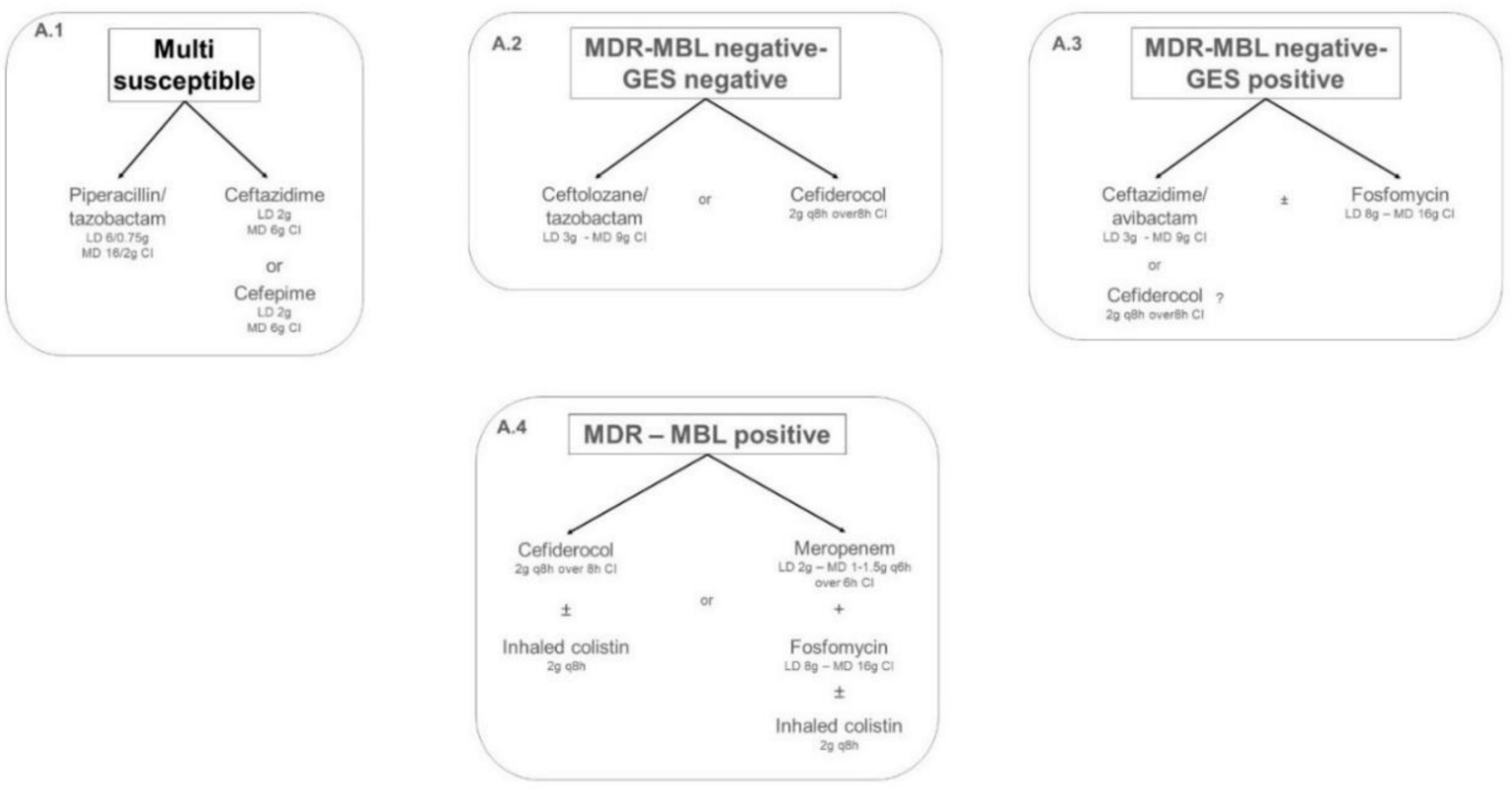

Figure 1. Algorithms for targeted treatment of IVAC caused by Pseudomonas aeruginosa with different pattern of antibiotic susceptibility. CI: continuous infusion; EI: extended infusion; LD: loading dose; MBL: metallo-beta-lactamase; MD: maintenance dose; MDR: multidrug resistance.

\subsubsection{Multi-Susceptible Pseudomonas aeruginosa}

Continuous infusion piperacillin-tazobactam (18 g/day after 6.75-9 g loading dose [LD]) is recommended as target therapy for the management of IVACs caused by multisusceptible Pseudomonas aeruginosa. Ceftazidime (6-8 g/day after 2 g LD) or cefepime ( $2 \mathrm{~g}$ LD followed by $6 \mathrm{~g} /$ day) in continuous infusion should be reserved as second-line alternatives in case of clinical or microbiological failure (Figure 1, panel A.1). ATS/IDSA guidelines [15] recommended the use of piperacillin-tazobactam, ceftazidime or cefepime as definitive therapy of HAP or VAP requiring a coverage on Pseudomonas aeruginosa with demonstrated susceptibility, suggesting the administration in EI or CI to maximize lung exposure. A summary of the studies evaluating the efficacy of piperacillin-tazobactam or third/fourth-generation cephalosporins in patients affected by IVACs caused by multisusceptible Pseudomonas aeruginosa is provided in Table 1. 
Table 1. Summary of the studies investigating the treatment of multi-susceptible Pseudomonas aeruginosa infection-related ventilator-associated complications (IVACs) with piperacillin-tazobactam or fourth-generation cephalosporins.

\begin{tabular}{|c|c|c|c|c|c|c|c|c|c|}
\hline $\begin{array}{l}\text { Author, Year } \\
\text { and } \\
\text { Reference }\end{array}$ & Study Design & No. of Patients & $\begin{array}{l}\text { Antibiotic and } \\
\text { Dosing }\end{array}$ & Rate of IVACs & Isolates & Severity & $\begin{array}{l}\text { Clinical } \\
\text { Outcomes }\end{array}$ & $\begin{array}{c}\text { Relapse Rate- } \\
\text { Resistance } \\
\text { Development }\end{array}$ & Comments \\
\hline \multicolumn{10}{|c|}{ Piperacillin-tazobactam } \\
\hline $\begin{array}{l}\text { Kalil et al., } \\
2016 \text { [15] }\end{array}$ & Guidelines & \multicolumn{8}{|c|}{$\begin{array}{c}\text { Piperacillin-tazobactam at dosage of } 4.5 \mathrm{~g} \text { q6h (preferring EI or CI) for empiric or definitive treatment of HAP/VAP caused by Pseudomonas aeruginosa according to antimicrobial susceptibility test (Strong } \\
\text { recommendation; low-quality evidence) }\end{array}$} \\
\hline $\begin{array}{l}\text { Jaccard et al., } \\
1998 \text { [16] }\end{array}$ & RCT, multicentre & $\begin{array}{l}371 \\
\text { (IMI } \\
\text { vs. } \\
\text { PIT) }\end{array}$ & $\begin{array}{l}\text { IMI } \\
500 \mathrm{mg} \mathrm{q6h} \\
\text { vs. } \\
\text { PIT } \\
4.5 \mathrm{~g} \mathrm{q} 8 \mathrm{~h}\end{array}$ & $49.2 \%$ HAP & $28 \%$ P. aeruginosa & $\begin{array}{l}\text { Mechanical } \\
\text { ventilation } 47 \% \\
\text { APACHE II score: } \\
14.6 \pm 6.8\end{array}$ & $\begin{array}{c}\text { Clinical failure rate: } \\
17 \% \text { (PIT) vs. } 29 \% \text { (IMI) } \\
p=0.09 \\
\text { Mortality rate for infection: } \\
8 \% \text { (PIT) vs. } 9 \% \text { (IMI) } \\
p=0.78 \\
\text { Clinical failure rate in } P . \\
\text { aeruginosa HAP: } \\
10 \% \text { (PIT) vs. } 50 \% \text { (IMI) } \\
p=0.004\end{array}$ & $\begin{array}{c}\text { Resistance } \\
\text { development } \\
\text { 25.0\% IMI vs. } 4.8 \% \\
\text { PIT }\end{array}$ & $\begin{array}{l}\text { PIT monotherapy is at least } \\
\text { as effective and safe as IMI } \\
\text { monotherapy in the } \\
\text { treatment of HAP. In } P \text {. } \\
\text { aeruginosa HAP, PIT } \\
\text { achieved a better clinical } \\
\text { efficacy than IMI, due to } \\
\text { reduced development of } \\
\text { microbiological resistance. }\end{array}$ \\
\hline $\begin{array}{l}\text { Joshi et al., } \\
1999 \text { [17] }\end{array}$ & $\begin{array}{c}\mathrm{RCT}, \\
\text { multicentre }\end{array}$ & $\begin{array}{c}300 \\
(155 \text { PIT + } \\
\text { tobramycin vs. } \\
145 \text { CTZ }+ \\
\text { tobramycin })\end{array}$ & $\begin{array}{c}\text { PIT } \\
3.375 \mathrm{~g} \mathrm{q} 4 \mathrm{~h} \\
+ \text { tobramycin } \\
5 \mathrm{mg} / \mathrm{kg} / \text { day } \\
\text { vs. } \\
\text { CTZ } \\
2 \mathrm{~g} \mathrm{q} 8 \mathrm{~h} \\
+ \text { tobramycin } \\
5 \mathrm{mg} / \mathrm{kg} / \text { day } \\
\end{array}$ & $87 \%$ HAP & $7.7 \%$ P. aeruginosa & Severe infection $21 \%$ & $\begin{array}{c}\text { Clinical cure rate: } \\
74.2 \% \text { (PIT) vs. } 57.9 \% \text { (CTZ) } \\
p=0.004 \\
\text { Clinical cure rate in } P . \\
\text { aeruginosa HAP: } \\
67 \% \text { (PIT) } \\
\text { vs. } 30 \% \text { (CTZ) } \\
p=\text { NS } \\
\end{array}$ & NA & $\begin{array}{l}\text { PIT plus tobramycin was } \\
\text { shown to be more effective } \\
\text { and as safe as CTZ plus } \\
\text { tobramycin in the treatment } \\
\text { of patients with HAP. A } \\
\text { trend to higher } \\
\text { microbiological eradication } \\
\text { was found } P \text {. aeruginosa } \\
\text { subgroup with PIT. }\end{array}$ \\
\hline $\begin{array}{l}\text { Babich et al., } \\
2020[18]\end{array}$ & $\begin{array}{l}\text { Retrospective, } \\
\text { multicentre, } \\
\text { propensity score } \\
\text { adjusted analysis }\end{array}$ & $\begin{array}{c}767 \\
\text { (213 CTZ } \\
\text { vs. } \\
210 \mathrm{MER} / \mathrm{IMI} \\
\text { vs. } \\
\text { 344 PIT) }\end{array}$ & $\begin{array}{c}\text { All } \\
\text { monotherapy } \\
\text { 83.3\% Intermittent } \\
\text { infusion }\end{array}$ & $\begin{array}{l}\text { All BSI } \\
14.7 \% \mathrm{HAP} / \mathrm{VAP}\end{array}$ & $\begin{array}{l}\text { 100\% P. aeruginosa } \\
\quad 7.6 \% \mathrm{MDR}\end{array}$ & $\begin{array}{c}\text { ICU admission } \\
16.6 \% \\
\text { Mechanical } \\
\text { ventilation } 12.1 \% \\
\text { SOFA score } 4(2-6)\end{array}$ & $\begin{array}{c}\text { Mortality rate: } \\
\text { 17.4\% (CTZ) vs. } 20 \% \text { (MER- } \\
\text { IMI) vs. } 16 \% \text { (PIT) } \\
p=\text { NS }\end{array}$ & $\begin{array}{c}\text { Resistance } \\
\text { development: } \\
17.5 \% \text { (MER-IMI) vs. } \\
12.4 \% \text { (CTZ) vs. } 8.4 \% \\
\text { (PIT) } \\
p=0.007\end{array}$ & $\begin{array}{l}\text { No significant difference in } \\
\text { mortality, clinical, and } \\
\text { microbiological outcomes } \\
\text { or adverse events was } \\
\text { demonstrated between } \\
\text { CTZ, carbapenems, and PIT } \\
\text { as definitive treatment of } P \text {. } \\
\text { aeruginosa bacteraemia. } \\
\text { Higher rates of resistance } \\
\text { development were found in } \\
\text { patients treated with } \\
\text { carbapenems. }\end{array}$ \\
\hline
\end{tabular}


Table 1. Cont.

\begin{tabular}{|c|c|c|c|c|c|c|c|c|c|}
\hline $\begin{array}{l}\text { Author, Year } \\
\text { and } \\
\text { Reference }\end{array}$ & Study Design & No. of Patients & $\begin{array}{l}\text { Antibiotic and } \\
\text { Dosing }\end{array}$ & Rate of IVACs & Isolates & Severity & $\begin{array}{l}\text { Clinical } \\
\text { Outcomes }\end{array}$ & $\begin{array}{l}\text { Relapse Rate- } \\
\text { Resistance } \\
\text { Development }\end{array}$ & Comments \\
\hline \multicolumn{10}{|c|}{ Third/fourth-generation cephalosporins (Ceftazidime-Cefepime) } \\
\hline $\begin{array}{l}\text { Kalil et al., } \\
2016 \text { [15] }\end{array}$ & Guidelines & \multicolumn{8}{|c|}{$\begin{array}{c}\text { Both ceftazidime and cefepime at dosage of } 2 \mathrm{~g} \text { q8h (preferring EI or CI) for empiric or definitive treatment of HAP/VAP caused by Pseudomonas aeruginosa according to antimicrobial susceptibility test } \\
\text { (strong recommendation; low-quality evidence) }\end{array}$} \\
\hline $\begin{array}{l}\text { Babich et al., } \\
2020 \text { [18] }\end{array}$ & $\begin{array}{l}\text { Retrospective, } \\
\text { multicentre, } \\
\text { propensity score } \\
\text { adjusted analysis }\end{array}$ & $\begin{array}{l}767 \\
\text { (213 CTZ } \\
\text { vs. } \\
\text { 210 MER/IMI vs. } \\
\text { 344 PIT) }\end{array}$ & $\begin{array}{c}\text { All } \\
\text { monotherapy } \\
\text { 83.3\% Intermittent } \\
\text { infusion }\end{array}$ & $\begin{array}{l}\text { All BSI } \\
14.7 \% \mathrm{HAP} / \mathrm{VAP}\end{array}$ & $\begin{array}{l}\text { 100\% P. aeruginosa } \\
7.6 \% \text { MDR }\end{array}$ & $\begin{array}{c}\text { ICU admission } \\
16.6 \% \\
\text { Mechanical } \\
\text { ventilation } 12.1 \% \\
\text { SOFA score } 4(2-6)\end{array}$ & $\begin{array}{c}\text { Mortality rate: } \\
\text { 17.4\% (CTZ) vs. } 20 \% \text { (MER- } \\
\text { IMI) vs. } 16 \% \text { (PIT) } \\
p=\text { NS }\end{array}$ & $\begin{array}{c}\text { Resistance } \\
\text { development: } \\
\text { 17.5\% (MER- } \\
\text { IMI) vs. } 12.4 \% \text { (CTZ) } \\
\text { vs. } 8.4 \% \text { (PIP-TZB) } \\
p=0.007\end{array}$ & $\begin{array}{l}\text { No difference in mortality } \\
\text { rate between ceftazidime } \\
\text { and carbapenems at } \\
\text { propensity score analysis } \\
\text { (OR 1.14; CI 0.52-2.46). } \\
\text { Significant higher } \\
\text { occurrence of new } \\
\text { resistance development in } P \\
\text { aeruginosa isolates in } \\
\text { patients treated with } \\
\text { carbapenems compared to } \\
\text { ceftazidime }(17.5 \% \text { vs. } \\
12.4 \% ; p=0.007) .\end{array}$ \\
\hline $\begin{array}{c}\text { Su et al., } 2017 \\
{[19]}\end{array}$ & Retrospective & 90 & $\begin{array}{l}\text { Cefepime } \\
2 \mathrm{~g} \text { q8h II }\end{array}$ & $\begin{array}{l}\text { All BSIs } \\
30 \% \text { HAP/VAP }\end{array}$ & $\begin{array}{l}\text { All P. aeruginosa } \\
\text { cefepime- } \\
\text { susceptible }\end{array}$ & $\begin{array}{c}\text { ICU admission } \\
32.2 \% \\
\text { Mechanical } \\
\text { ventilation } 25.6 \% \\
\text { Severe sepsis/septic } \\
\text { shock 23.3\% } \\
\text { Mean APACHE II } \\
\text { score: } 22.07 \\
\text { Neutropenia 20\% }\end{array}$ & $\begin{array}{l}\text { Overall 30-day mortality } \\
\text { rate: } \\
36.7 \% \\
\text { Overall 30-day mortality } \\
\text { rate in HAP /VAP } \\
\text { subgroup: } 59.3 \%\end{array}$ & NA & $\begin{array}{l}\text { A cefepime MIC of } 4 \mathrm{mg} / \mathrm{L} \\
\text { may predict an } \\
\text { unfavourable outcome } \\
\text { among patients with } \\
\text { serious infections caused by } \\
\text { P. aeruginosa. }\end{array}$ \\
\hline $\begin{array}{l}\text { Ratliff et al., } \\
2017 \text { [20] }\end{array}$ & $\begin{array}{l}\text { Retrospective, } \\
\text { propensity score } \\
\text { matched analysis }\end{array}$ & $\begin{array}{c}58 \\
(29 \mathrm{MIC} \leq 2 \mathrm{mg} / \mathrm{L} \\
\text { vs. } 29 \mathrm{MIC}> \\
2 \mathrm{mg} / \mathrm{L})\end{array}$ & $\begin{array}{l}\text { Ceftazidime } \\
2 \mathrm{~g} \mathrm{q} 8 \mathrm{~h} \\
\text { or } \\
\text { Cefepime } \\
2 \mathrm{~g} \mathrm{q} 12 \mathrm{~h}\end{array}$ & $\begin{array}{l}\text { All BSIs } \\
22.4 \% \mathrm{HAP} / \mathrm{VAP}\end{array}$ & All $P$. aeruginosa & NA & $\begin{array}{c}\text { 30-day } \\
\text { mortality rate: } \\
17.2 \% \text { vs. } 27.6 \%(p=0.34)\end{array}$ & NA & $\begin{array}{l}\text { No subgroup analysis was } \\
\text { performed according to site } \\
\text { of infection. }\end{array}$ \\
\hline
\end{tabular}

BSI: bloodstream infections; CTZ: ceftazidime; CI: continuous infusion; EI: extended infusion; HAP: hospital-acquired pneumonia; ICU: intensive care unit; IMI: imipenem-cilastatin MER: meropenem; MIC: minimum inhibitory concentration NA: not available; OR: odds ratio; PIT: piperacillin-tazobactam; RCT: randomized controlled trial; VAP: ventilator-associated pneumonia. 
Two different RCTs investigated the efficacy of piperacillin-tazobactam in the management of IVACs caused by Pseudomonas aeruginosa [16,17]. Jaccard et al. [16] randomized 371 patients affected by nosocomial infections ( $49.2 \%$ HAP, of which $28 \%$ caused by Pseudomonas aeruginosa) to piperacillin-tazobactam or imipenem-cilastatin. Although no difference in clinical failure rate $(17.0 \%$ vs. $29.0 \% ; p=0.09)$ and mortality rate $(8 \%$ vs. $9 \% ; p=0.78$ ) was found between the two agents in HAP subgroup, a significantly lower clinical failure rate was found in patients affected by HAP due to Pseudomonas aeruginosa receiving piperacillin-tazobactam compared to imipenem-cilastatin $(10.0 \%$ vs. $50.0 \%$; $p=0.004)$. Notably, a significantly higher rate of resistance development was reported with imipenem-cilastatin compared to piperacillin-tazobactam in HAP due to Pseudomonas aeruginosa. Joshi et al. [17] randomized 300 patients (87\% HAP, of which $7.7 \%$ due to Pseudomonas aeruginosa), reporting a significantly higher clinical cure rate in 155 patients receiving piperacillin-tazobactam associated with tobramycin compared to 145 patients treated with ceftazidime plus tobramycin $(74.2 \%$ vs. $57.9 \% ; p=0.004)$. Furthermore, a trend to higher microbiological eradication was found in Pseudomonas aeruginosa subgroup with piperacillin-tazobactam (67\% vs. 30\%; $p=0.19)$. Babich et al. [18] retrospectively evaluated clinical outcome in 767 patients receiving definitive monotherapy for the treatment of BSI due to Pseudomonas aeruginosa. No difference in 30-day mortality rate emerged between ceftazidime (OR 1.14; 95\% CI 0.52-2.46) or piperacillin-tazobactam (OR 1.30; 95\% CI 0.67-2.51) compared to carbapenems (meropenem or imipenem) at propensity score analysis, while a higher development of new resistance in Pseudomonas aeruginosa isolates occurred in patients treated with carbapenems compared to ceftazidime or piperacillin-tazobactam ( $17.5 \%$ vs. $12.4 \%$ vs. $8.4 \% ; p=0.007)$. However, no subgroup analysis according to IVACs was performed.

Several observational studies recently investigated the role of ceftazidime and cefepime in the management of Pseudomonas aeruginosa BSIs [18-20]. Rate of IVACs ranged from $14.7 \%$ to $30 \%$ and ICU admission was reported in up to $32.2 \%$ of cases. Conflicting findings emerged in retrospective studies performing a subgroup analysis according to MIC of Pseudomonas aeruginosa for ceftazidime or cefepime [19,20]. Su et al. [19] found that a MIC for cefepime $\geq 4 \mathrm{mg} / \mathrm{L}$ may predict an unfavourable outcome among patients with serious infections due to Pseudomonas aeruginosa, including 30\% of HAP/VAP. Conversely, Ratliff et al. [20] found no difference in mortality rate between patients exhibiting a MIC for ceftazidime or cefepime $\leq 2 \mathrm{mg} / \mathrm{L}$ compared to those with a MIC $>2 \mathrm{mg} / \mathrm{L}$ in Pseudomonas aeruginosa BSIs $(17.2 \%$ vs. $27.6 \% ; p=0.34)$, although subgroup analysis in subjects affected by IVACs was not performed.

In regard to recommended dosages, some well-established evidence may support the use of CI over intermittent infusion in administering traditional antipseudomonal beta-lactams in critically ill patients [21]. Additionally, we recently showed in a large cohort of critically ill patients having documented Gram-negative infections treated with CI traditional beta-lactams that early achievement of an aggressive PK/PD target of Css/MIC > 5 within the first $72 \mathrm{~h}$ was significantly associated with both microbiological eradication and prevention of resistance development [22]. In the same study, Pseudomonas aeruginosa-related infections were independently associated with higher risk of microbiological failure [22]. Accordingly, we consider that when treating IVACs caused by multi-susceptible Pseudomonas aeruginosa isolates, the use of CI piperacillin-tazobactam and/or ceftazidime, and/or cefepime after loading may represent a valuable approach for rapidly achieving and maintaining an aggressive PK/PD target helpful at achieving microbiological eradication.

\subsubsection{Multidrug-Resistant (MDR) Metallo-Beta-Lactamase (MBL)-Negative Pseudomonas aeruginosa}

The emergence of MDR and extensively drug-resistant (XDR) Pseudomonas aeruginosa is a major clinical concern. The underlying mechanisms of the MDR/XDR phenotype are heterogeneous and can be mediated by the selection of mutations in the chromosomal genes or by horizontal acquisition of resistance determinants, including beta-lactamase and 
carbapenemase genes [23]. A major distinction regards MDR/XDR Pseudomonas aeruginosa isolates producing or not metallo-beta-lactamases. In case of MDR/XDR metallo-betalactamases (MBL)-negative isolates, antibiotics should be chosen according to antimicrobial susceptibility tests. Among the different beta-lactamases responsible for MDR/XDR phenotype, GES enzymes, belonging to class A carbapenemases, could play a remarkable role in the selection of appropriate targeted anti-pseudomonal agents. GES enzymes represents a major resistance mechanism of MDR/XDR high-risk clones (e.g., ST-235 highly virulent clone), exhibiting higher virulence compared to other clones (e.g., ST111 or ST175) and resulting in more severe acute infections with significant mortality [23]. Furthermore, production of GES enzymes confers resistance to ceftolozane-tazobactam [23]. In case of IVACs caused by MDR/XDR MBL-negative GES-negative isolates (Figure 1, Panel A.2), prolonged infusion of ceftolozane-tazobactam ( $3 \mathrm{~g}$ q8h CI after $3 \mathrm{~g} \mathrm{LD}$ ) is recommended as first-line therapy. Cefiderocol ( $2 \mathrm{~g}$ LD followed by $2 \mathrm{~g}$ q8h in CI) should be reserved as second-line alternative in case of clinical or microbiological failure (Figure 1, Panels A.2-A.3). A summary of the studies evaluating the efficacy of ceftolozane-tazobactam or cefiderocol in patients affected by IVACs caused by MDR GES-negative Pseudomonas aeruginosa is provided in Table 2 .

In a phase III RCT, Kollef et al. [24] reported no significant difference in mortality (25.4\% vs. $18.5 \% ; p=$ NS) and clinical cure rate (57.1\% vs. $60 \%)$ between ceftolozanetazobactam and meropenem in subgroup of critically ill patients affected by HAP or VAP caused by MDR/XDR Pseudomonas aeruginosa. However, Pseudomonas aeruginosa accounted only for $17.4 \%$ of overall isolates. Several observational studies and case series [25-40] reported the high efficacy of ceftolozane-tazobactam in critically ill patients (ICU admission ranging from $23.8 \%$ to $100 \%$ ) affected by HAP/VAP in most cases, although both relapse and resistance rate were not negligible (respectively up 29\% and 14\%). Notably, Pogue et al. [25] retrospectively analysed two hundred MDR Pseudomonas aeruginosa (64\% HAP/VAP) infections, 100 treated with ceftolozane-tazobactam and the other 100 managed with polymyxin- or aminoglycoside-based regimen. A significant higher clinical cure rate $(81 \%$ vs. $61 \% ; p=0.002)$ and lower AKI occurrence $(6 \%$ vs. $34 \% ; p<0.001)$ was found in patients treated with ceftolozane-tazobactam. Similarly, Fernandez-Cruz et al. [28] found a significant lower mortality rate in 19 patients treated with ceftolozanetazobactam compared to 38 subjects receiving other anti-pseudomonal agents $(5.4 \%$ vs. $28.9 \% ; p=0.045$ ) for infections caused by ST-175 Pseudomonas aeruginosa clones. All patients were affected by haematological malignancies and rate of nosocomial pneumonia was 26.3\%. Interestingly, Gallagher et al. [29] retrospectively analysed 205 patients affected by severe Pseudomonas aeruginosa infection (51.2\% ICU admission; 59\% HAP/VAP) and treated with ceftolozane-tazobactam, reporting that pneumonia was significantly associated with lower microbiological cure than observed for other infection sites (OR 0.12; 95\% CI 0.05-0.30). Rodriguez-Nunez et al. [30] assessed 90 critically ill patients affected by lower respiratory tract infections due to MDR/XDR Pseudomonas aeruginosa treated with ceftolozane-tazobactam, founding that a MIC $>2 \mathrm{mg} / \mathrm{L}$ was an independent predictor of mortality at multivariate analysis.

Two different phase III RCTs [41,42] supported the efficacy of cefiderocol for the treatment of IVACs caused MDR Pseudomonas aeruginosa, reporting no significant difference in mortality or clinical cure rate compared to meropenem or best available therapy (including combination of aminoglycoside, carbapenems, colistin, fosfomycin or tigecycline) in case of carbapenem-resistant isolates. However, the proportion of carbapenemase-producer isolates in these studies was only $8-15 \%$. Several in vitro studies [43-45] demonstrated the activity of cefiderocol against MDR (carbapenemase-negative meropenem non-susceptible) Pseudomonas aeruginosa (MIC range 0.002-8; MIC50 0.12). Notably, high susceptibility (90\%) against GES-positive isolates was reported. 
Table 2. Summary of the studies investigating the treatment of multidrug-resistant (MDR) metallo-beta-lactamase-negative GES-negative Pseudomonas aeruginosa infection-related ventilator-associated complications (IVACs) with ceftolozane-tazobactam or cefiderocol.

\begin{tabular}{|c|c|c|c|c|c|c|c|c|c|}
\hline $\begin{array}{l}\text { Author, Year } \\
\text { and Reference }\end{array}$ & Study Design & No. of Patients & $\begin{array}{l}\text { Antibiotic and } \\
\text { Dosing }\end{array}$ & Rate of IVACs & Isolates & Severity & $\begin{array}{l}\text { Clinical } \\
\text { Outcomes }\end{array}$ & $\begin{array}{c}\text { Relapse Rate- } \\
\text { Resistance } \\
\text { Development }\end{array}$ & Comments \\
\hline \multicolumn{10}{|c|}{ Ceftolozane-tazobactam } \\
\hline $\begin{array}{l}\text { Kollef et al., } \\
2019 \text { [24] }\end{array}$ & $\begin{array}{l}\text { phase III RCT, } \\
\text { multicentre } \\
\text { (ASPECT-NP) }\end{array}$ & $\begin{array}{c}726 \\
\text { (362 CTT vs. } 364 \\
\text { MER) }\end{array}$ & $\begin{array}{l}\text { CTT } \\
3 \mathrm{~g} \mathrm{q} 8 \mathrm{~h} \\
\text { vs. } \\
\text { MER } \\
1 \mathrm{~g} \mathrm{q} 8 \mathrm{~h}\end{array}$ & $\begin{array}{l}\text { All nosocomial } \\
\text { pneumonia } \\
71 \% \text { VAP } \\
6 \% \text { secondary BSI }\end{array}$ & $\begin{array}{l}\text { 17.4\% P. aeruginosa } \\
\text { 38.1\% MDR-PA } \\
\text { 15.9\% XDR-PA }\end{array}$ & $\begin{array}{c}\text { ICU admission } 92 \% \\
\text { APACHE II score } \geq 2033 \% \\
\text { Median SOFA score } 6 \\
\text { Median CPIS } 10 \\
\text { Median duration of } \\
\text { mechanical ventilation: } 5 \\
\text { days }\end{array}$ & $\begin{array}{c}\text { 28-day mortality rate in } P . \\
\text { aeruginosa subgroup: } \\
25.4 \% \text { vs. } 18.5 \% \\
(p=\text { NS) } \\
\text { Clinical cure rate } \\
57.1 \% \text { vs. } 60 \% \\
\text { ( } p=\mathrm{NS})\end{array}$ & NA & $\begin{array}{c}\text { High-dose CTT is an } \\
\text { efficacious and well } \\
\text { tolerated treatment for } \\
\text { Gram-negative HAP/VAP } \\
\text { No difference in mortality } \\
\text { and clinical cure rate for } P \text {. } \\
\text { aeruginosa between CTT } \\
\text { and MER, including MDR } \\
(54.2 \% \text { vs. } 54.5 \%) \text { and XDR } \\
\text { isolates }(40 \% \text { vs. } 40 \%)\end{array}$ \\
\hline $\begin{array}{l}\text { Pogue et al., } \\
2019 \text { [25] }\end{array}$ & $\begin{array}{l}\text { Retrospective } \\
\text { observational } \\
\text { comparative, } \\
\text { multicentre }\end{array}$ & $\begin{array}{l}200 \\
\text { (100 LOZ-TAZ vs. } \\
100 \text { polymyxin- or } \\
\text { aminoglycoside- } \\
\text { combination } \\
\text { therapy) }\end{array}$ & $\begin{array}{c}\text { CTT } \\
\text { 1.5-3 g q8h } \\
\text { vs. } \\
\text { COL/polymyxin B } \\
\text { or } \\
\text { gentamycin- } \\
\text { amikacin-- } \\
\text { tobramycin }\end{array}$ & $\begin{array}{l}52 \% \text { VAP } \\
12 \% \text { HAP }\end{array}$ & $100 \%$ P. aeruginosa & $\begin{array}{c}\text { ICU admission } 69 \% \\
\text { Mechanical ventilation } \\
63 \% \\
\text { Severe } \\
\text { sepsis/septic shock } 42 \% \\
\text { Immunosuppression } 14 \%\end{array}$ & $\begin{array}{c}\text { Clinical cure rate: } \\
81 \% \text { vs. } 61 \% \\
(p=0.002) \\
\text { Overall AKI rate: } \\
6 \% \text { vs. } 34 \% \\
(p<0.001) \\
\text { In-hospital mortality rate: } \\
20 \% \text { vs. } 25 \% \\
(p=0.40)\end{array}$ & $\begin{array}{c}\text { Relapse } \\
14 \% \text { vs. } 16 \% \\
\text { ( } p=\text { NS) }\end{array}$ & $\begin{array}{c}\text { CTT was independently } \\
\text { associated with clinical } \\
\text { cure (OR } 2.63 ; 95 \% \text { CI } \\
1.31-5.30 \text { ) and protective } \\
\text { against AKI (OR } 0.08 ; 95 \% \\
\text { CI 0.03-0.22) } \\
\text { Preferential use CTT over } \\
\text { polymyxins or } \\
\text { aminoglycosides for } \\
\text { MDR-PA infections. }\end{array}$ \\
\hline $\begin{array}{l}\text { Bassetti et al., } \\
2019 \text { [26] }\end{array}$ & $\begin{array}{l}\text { Retrospective } \\
\text { observational, } \\
\text { multicentre } \\
\text { (CEFTABUSE) }\end{array}$ & 101 & $\begin{array}{c}\text { CTT } \\
\text { 1.5-3 g q8h } \\
\text { (CI/EI 18.8\%) } \\
\text { 38.6\% first-line } \\
\text { therapy }\end{array}$ & $31.7 \%$ HAP/VAP & $\begin{array}{c}\text { 100\% P. aeruginosa } \\
\text { 17.8\% MDR-PA } \\
50.5 \% \text { XDR-PA } \\
\text { 2\% PDR-PA }\end{array}$ & $\begin{array}{c}\text { ICU admission } 23.8 \% \\
\text { Mechanical ventilation } \\
18.8 \% \\
\text { Septic shock } 11.9 \% \\
\text { Solid organ transplant } \\
\text { recipients } 10.9 \% \\
\text { Haematological } \\
\text { malignancy } 12.9 \% \\
\text { Neutropenia } 10.9 \% \\
\end{array}$ & $\begin{array}{c}\text { Clinical cure rate: } \\
83.2 \%\end{array}$ & $\begin{array}{c}\text { Relapse 7\% } \\
\text { Resistance 3\% }\end{array}$ & $\begin{array}{l}\text { Lower clinical success in } \\
\text { patients with sepsis or } \\
\text { requiring CRRT. } \\
\text { Higher clinical failure } \\
\text { (25.0) in pneumonia } \\
\text { subgroup compared to } \\
\text { other types of infection }\end{array}$ \\
\hline $\begin{array}{l}\text { Balandin et al., } \\
2020[27]\end{array}$ & $\begin{array}{l}\text { Retrospective } \\
\text { observational, } \\
\text { multicentre }\end{array}$ & 95 & $\begin{array}{c}\text { CTT } \\
1.5-3 \mathrm{~g} \mathrm{q} 8 \mathrm{~h}\end{array}$ & $\begin{array}{c}56.2 \% \mathrm{HAP} / \mathrm{VAP} \\
8.4 \% \mathrm{VAT}\end{array}$ & $\begin{array}{c}100 \% \text { P. aeruginosa } \\
\text { 48.4\% XDR-PA } \\
36.8 \% \text { MDR-PA }\end{array}$ & $\begin{array}{c}\text { ICU admission } 100 \% \\
\text { Mechanical ventilation } \\
80 \% \\
\text { Septic shock } 45.3 \% \\
\text { RRT 27.4\% } \\
\text { Mean SOFA } 6.9 \\
\text { Solid organ transplant } \\
\text { recipients } 6.2 \%\end{array}$ & $\begin{array}{l}\text { Microbiological } \\
\text { eradication: } \\
42.1 \% \\
\text { ICU mortality: } \\
36.5 \%\end{array}$ & Relapse $22.9 \%$ & $\begin{array}{l}\text { Mortality rate in } \\
\text { pneumonia subgroup: } \\
34 \%\end{array}$ \\
\hline
\end{tabular}


Table 2. Cont.

\begin{tabular}{|c|c|c|c|c|c|c|c|c|c|}
\hline $\begin{array}{l}\text { Author, Year } \\
\text { and Reference }\end{array}$ & Study Design & No. of Patients & $\begin{array}{l}\text { Antibiotic and } \\
\text { Dosing }\end{array}$ & Rate of IVACs & Isolates & Severity & $\begin{array}{l}\text { Clinical } \\
\text { Outcomes }\end{array}$ & $\begin{array}{l}\text { Relapse Rate- } \\
\text { Resistance } \\
\text { Development }\end{array}$ & Comments \\
\hline $\begin{array}{l}\text { Fernandez-Cruz } \\
\text { et al., } 2019[28]\end{array}$ & $\begin{array}{l}\text { Retrospective } \\
\text { case-control }\end{array}$ & $\begin{array}{c}57 \\
\text { (19 CTT } \\
\text { vs. } 38 \text { other agents) }\end{array}$ & $\begin{array}{c}\text { CTT } \\
3 \mathrm{~g} \mathrm{~g} \text { gh } \\
\text { (HAP/VAP or BSI) } \\
\text { 84.6\% targeted } \\
\text { therapy }\end{array}$ & $26.3 \% \mathrm{HAP} / \mathrm{VAP}$ & $\begin{array}{l}100 \% \text { P. aeruginosa } \\
\\
52.6 \% \text { MDR-PA } \\
47.4 \% \text { XDR-PA } \\
100 \% \text { ST- }-175 \text { clone }\end{array}$ & $\begin{array}{c}\text { ICU admission } 26.3 \% \\
\text { Haematological } \\
\text { malignancy } 100 \% \\
\text { Neutropenia } 63.2 \% \\
\text { Sepsis } 15.8 \% \\
\text { Mean SOFA } 5.42\end{array}$ & $\begin{array}{c}\text { 14-day clinical cure rate: } \\
89.5 \% \text { vs. } 71.1 \% \\
(p=0.18) \\
\text { 30-day mortality rate: } \\
5.4 \% \text { vs. } 28.9 \% \\
(p=0.045)\end{array}$ & $\begin{array}{l}\text { Relapse } \\
15.8 \%\end{array}$ & $\begin{array}{c}\text { CTT } \\
\text { showed lower mortality } \\
\text { compared to traditional } \\
\text { therapy in severe PA } \\
\text { infections in } \\
\text { haematological patients. } \\
\text { No subgroup analysis in } \\
\text { patients with HAP/VAP } \\
\text { was performed. }\end{array}$ \\
\hline $\begin{array}{l}\text { Gallagher et al., } \\
2018 \text { [29] }\end{array}$ & $\begin{array}{l}\text { Retrospective } \\
\text { observational, } \\
\text { multicentre }\end{array}$ & 205 & $\begin{array}{c}\text { CTT } \\
1.5-3 \mathrm{~g} \text { q8h } \\
\text { Dose adjustment } \\
\text { according to renal } \\
\text { function }\end{array}$ & $59 \% \mathrm{HAP} / \mathrm{VAP}$ & $100 \%$ P. aeruginosa & $\begin{array}{c}\text { ICU admission } 51.2 \% \\
\text { Median APACHE II score } \\
19 \\
\text { Solid organ transplant } \\
\text { recipients } 17.1 \%\end{array}$ & $\begin{array}{l}\text { Overall mortality rate: } \\
19 \% \\
\text { Clinical cure rate: } \\
73.7 \%\end{array}$ & NA & $\begin{array}{c}\text { Mortality rate was higher } \\
\text { in VAP subgroup (37.9\% } \\
\text { vs. 19\%) } \\
\text { Clinical success was lower } \\
\text { in VAP subgroup (50\% vs. } \\
73.7 \% \text { ) } \\
\text { Pneumonia was associated } \\
\text { with significant lower } \\
\text { microbiological cure (OR } \\
0.12 ; 95 \% \text { CI } 0.05-0.30)\end{array}$ \\
\hline $\begin{array}{l}\text { Rodriguez- } \\
\text { Nunez et al., } \\
2019 \text { [30] }\end{array}$ & $\begin{array}{l}\text { Retrospective } \\
\text { observational, } \\
\text { multicentre }\end{array}$ & 90 & $\begin{array}{c}\text { CTT } \\
60 \% 3 \mathrm{~g} \mathrm{q} 8 \mathrm{~h}\end{array}$ & $\begin{array}{c}70 \% \text { HAP /VAP } \\
30 \% \text { VAT }\end{array}$ & $\begin{array}{c}\text { 76.7\% XDR-PA } \\
23.3 \% \text { MDR-PA } \\
\text { Median MIC } 2 \\
\text { mg/L }\end{array}$ & $\begin{array}{c}\text { Septic shock } 34.4 \% \\
\text { RRT } 12.2 \% \\
\text { Solid organ transplant } \\
\text { recipients } 8.9 \%\end{array}$ & $\begin{array}{l}\text { 30-day } \\
\text { mortality rate: } \\
27.8 \%\end{array}$ & NA & $\begin{array}{l}\mathrm{MIC}>2 \mathrm{mg} / \mathrm{L} \text { was an } \\
\text { independent predictor of } \\
\text { mortality at multivariate } \\
\text { analysis }\end{array}$ \\
\hline $\begin{array}{l}\text { Munita et al., } \\
2017 \text { [31] }\end{array}$ & $\begin{array}{l}\text { Retrospective } \\
\text { observational }\end{array}$ & 35 & $\begin{array}{c}\text { CTT } \\
1.5-3 \mathrm{~g} \text { q8h } \\
\text { Dose adjustment } \\
\text { according to renal } \\
\text { function }\end{array}$ & $51 \% \mathrm{HAP} / \mathrm{VAP}$ & $100 \%$ CR-PA & NA & $\begin{array}{l}\text { Overall clinical cure rate: } \\
\qquad 74 \%\end{array}$ & NA & $\begin{array}{l}38.9 \% \text { clinical failure rate } \\
\text { in HAP/VAP subgroup }\end{array}$ \\
\hline $\begin{array}{l}\text { Haidar et al., } \\
2017 \text { [32] }\end{array}$ & $\begin{array}{l}\text { Retrospective } \\
\text { observational }\end{array}$ & 21 & $\begin{array}{c}\text { CTT } \\
1.5-3 \mathrm{~g} \mathrm{q} 8 \mathrm{~h}\end{array}$ & 85.7\% HAP/VAP & 100\% MDR-PA & $\begin{array}{c}\text { Mechanical ventilation } \\
38 \% \\
\text { Immunosuppression } 43 \%\end{array}$ & $\begin{array}{l}\text { 30-day mortality rate: } \\
10 \% \\
\text { Clinical failure rate: } \\
29 \%\end{array}$ & $\begin{array}{c}\text { Relapse 29\% } \\
\text { Resistance 14\% }\end{array}$ & $\begin{array}{l}33.3 \% \text { clinical failure rate } \\
\text { in HAP/VAP subgroup }\end{array}$ \\
\hline $\begin{array}{l}\text { Bosaeed et al., } \\
2020 \text { [33] }\end{array}$ & $\begin{array}{l}\text { Retrospective } \\
\text { observational }\end{array}$ & 19 & $\begin{array}{c}\text { CTT } \\
1.5-3 \mathrm{~g} \mathrm{q} 8 \mathrm{~h}\end{array}$ & $\begin{array}{l}16 \% \text { HAP } \\
16 \% \text { VAP }\end{array}$ & $100 \%$ CR-PA & $\begin{array}{l}\text { ICU admission } 63 \% \\
\text { Haematological } \\
\text { malignancy } 26 \%\end{array}$ & $\begin{array}{c}\text { 30-day mortality rate: } \\
21 \% \\
\text { Microbiological } \\
\text { eradication: } \\
74 \%\end{array}$ & NA & $\begin{array}{l}\text { Microbiological failure in } \\
50 \% \text { of HAP/VAP cases }\end{array}$ \\
\hline
\end{tabular}


Table 2. Cont.

\begin{tabular}{|c|c|c|c|c|c|c|c|c|c|}
\hline $\begin{array}{l}\text { Author, Year } \\
\text { and Reference }\end{array}$ & Study Design & No. of Patients & $\begin{array}{l}\text { Antibiotic and } \\
\text { Dosing }\end{array}$ & Rate of IVACs & Isolates & Severity & $\begin{array}{l}\text { Clinical } \\
\text { Outcomes }\end{array}$ & $\begin{array}{l}\text { Relapse Rate- } \\
\text { Resistance } \\
\text { Development }\end{array}$ & Comments \\
\hline \multirow{2}{*}{$\begin{array}{c}\text { Diaz } \\
\text {-Canestro et al., } \\
2018 \text { [34] }\end{array}$} & \multirow{2}{*}{$\begin{array}{l}\text { Prospective } \\
\text { observational }\end{array}$} & \multirow{2}{*}{58} & $\begin{array}{c}\text { CTT } \\
1.5-3 \mathrm{~g} \mathrm{q} 8 \mathrm{~h}\end{array}$ & \multirow{2}{*}{$60.3 \% \mathrm{HAP} / \mathrm{VAP}$} & \multirow{2}{*}{$\begin{array}{c}\text { 86.2\% XDR-PA } \\
\text { 10.3\% MDR-PA } \\
50 \% \text { ST-175 clone }\end{array}$} & \multirow{2}{*}{$\begin{array}{c}\text { ICU admission } 27.6 \% \\
\text { Mechanical ventilation } \\
32.8 \% \\
\text { Immunosuppression } \\
12.1 \% \\
\text { Median SOFA } 3\end{array}$} & \multirow{2}{*}{$\begin{array}{l}\text { Clinical cure rate: } \\
63.8 \% \\
\text { 30-day mortality rate: } \\
27.6 \%\end{array}$} & \multirow{2}{*}{ Resistance $13.8 \%$} & $\begin{array}{l}\text { Clinical failure was } \\
\text { documented in } 42.9 \% \text { of } \\
\text { HAP/VAP }\end{array}$ \\
\hline & & & $\begin{array}{l}91.4 \% \text { targeted } \\
\text { therapy }\end{array}$ & & & & & & $\begin{array}{l}\text { ST-175 clone associated } \\
\text { with higher risk of clinical } \\
\text { failure at multivariate } \\
\text { analysis }\end{array}$ \\
\hline $\begin{array}{l}\text { Escola-Verge } \\
\text { et al., 2018 [35] }\end{array}$ & $\begin{array}{l}\text { Retrospective } \\
\text { observational }\end{array}$ & 38 & $\begin{array}{c}\text { CTT } \\
1.5-3 \mathrm{~g} \mathrm{q} 8 \mathrm{~h}\end{array}$ & $36.8 \% \mathrm{HAP} / \mathrm{VAP}$ & $\begin{array}{c}\text { 100\% XDR-PA } \\
\text { Median CTT MIC: } \\
2 \text { mg/L }\end{array}$ & $\begin{array}{c}\text { ICU admission } 31.6 \% \\
\text { Solid organ transplant } \\
\text { recipients } 28.9 \% \\
\text { Neutropenia } 15.8 \%\end{array}$ & $\begin{array}{c}\text { 90-day clinical cure: } \\
68.4 \% \\
\text { 90-day mortality rate: } \\
13.2 \%\end{array}$ & Relapse $21.1 \%$ & $\begin{array}{l}\text { Clinical failure in } \\
\text { HAP/VAP subgroup: } 25 \%\end{array}$ \\
\hline $\begin{array}{l}\text { Xipell et al., } \\
2018 \text { [36] }\end{array}$ & $\begin{array}{l}\text { Retrospective } \\
\text { observational }\end{array}$ & 23 & CTT & $\begin{array}{l}\text { 17.4\% HAP } \\
17.4 \% \text { VAT }\end{array}$ & $\begin{array}{c}79 \% \text { XDR-PA } \\
17 \% \text { MDR-PA } \\
4 \% \text { PDR-PA }\end{array}$ & NA & $\begin{array}{c}\text { Clinical cure rate: } \\
87.5 \% \\
\text { 6-weeks mortality rate: } \\
21.7 \%\end{array}$ & NA & $\begin{array}{l}\text { Higher mortality rate in } \\
\text { respiratory tract infections } \\
(37 \%) . \\
\text { Significant higher } \\
\text { mortality rate in patients } \\
\text { with HAP/VAP treated } \\
\text { with low-dosage }(1.5 \mathrm{~g} \\
\text { q8h) vs. high-dose }(3 \mathrm{~g} \\
\text { q8h) CTT }(60 \% \text { vs. } 0 \%)\end{array}$ \\
\hline $\begin{array}{l}\text { Caston et al., } \\
2017[37]\end{array}$ & Case series & 12 & $\begin{array}{l}\text { CTT } \\
100 \% \text { targeted } \\
\text { therapy }\end{array}$ & $50 \% \mathrm{HAP} / \mathrm{VAP}$ & $100 \%$ MDR-PA & Septic shock $83.3 \%$ & $\begin{array}{l}\text { Overall mortality rate: } \\
25 \% \\
\text { Microbiological } \\
\text { eradication: } \\
83.3 \%\end{array}$ & Resistance: $16.6 \%$ & $\begin{array}{c}\text { Mortality rate in } \\
\text { HAP/VAP subgroup: } \\
33.3 \%\end{array}$ \\
\hline $\begin{array}{c}\text { Dinh et al., } 2017 \\
\text { [38] }\end{array}$ & Case series & 15 & $\begin{array}{c}\text { CTT } \\
\text { Median daily dose } \\
6 \mathrm{~g} / \text { day }\end{array}$ & $\begin{array}{c}46.7 \% \text { nosocomial } \\
\text { pneumonia }(85.7 \% \\
\text { VAP) }\end{array}$ & $100 \%$ XDR-PA & $\begin{array}{c}\text { ICU admission } 53.3 \% \\
\text { Mean SOFA score } 7.6 \\
\text { Immunosuppression } \\
66.7 \%\end{array}$ & $\begin{array}{c}\text { Clinical failure: } \\
33.3 \% \\
\text { In-hospital mortality rate: } \\
27 \% \\
\end{array}$ & Relapse $11.1 \%$ & $\begin{array}{l}\text { Clinical failure in } \\
\text { nosocomial pneumonia } \\
\text { subgroup: } 40 \%\end{array}$ \\
\hline $\begin{array}{l}\text { Gelfand et al., } \\
2015[39]\end{array}$ & Case series & 3 & $\begin{array}{c}\text { CTT } \\
3 \mathrm{gq} 8 \mathrm{~h}\end{array}$ & $100 \%$ VAP & $100 \%$ MDR-PA & $\begin{array}{l}\text { ICU admission } 100 \% \\
\text { Mechanical ventilation } \\
100 \%\end{array}$ & $\begin{array}{l}\text { Clinical cure: } \\
100 \%\end{array}$ & NA & \\
\hline $\begin{array}{l}\text { Hakki et al., } \\
2018 \text { [40] }\end{array}$ & Case series & 6 & $\begin{array}{c}\text { CTT } \\
3 \mathrm{~g} \mathrm{q} 8 \mathrm{~h}\end{array}$ & $50 \%$ pneumonia & $100 \%$ MDR-PA & $\begin{array}{c}\text { Haematopoietic-cell } \\
\text { transplant recipients } \\
100 \%\end{array}$ & $\begin{array}{c}\text { Clinical cure rate: } \\
\quad 66.7 \%\end{array}$ & Relapse $28.6 \%$ & $\begin{array}{l}\text { 33.3\% clinical failure rate } \\
\text { in patients with } \\
\text { nosocomial pneumonia }\end{array}$ \\
\hline
\end{tabular}


Table 2. Cont.

\begin{tabular}{|c|c|c|c|c|c|c|c|c|c|}
\hline $\begin{array}{l}\text { Author, Year } \\
\text { and Reference }\end{array}$ & Study Design & No. of Patients & $\begin{array}{l}\text { Antibiotic and } \\
\text { Dosing }\end{array}$ & Rate of IVACs & Isolates & Severity & $\begin{array}{l}\text { Clinical } \\
\text { Outcomes }\end{array}$ & $\begin{array}{c}\text { Relapse Rate- } \\
\text { Resistance } \\
\text { Development }\end{array}$ & Comments \\
\hline \multicolumn{10}{|c|}{ Cefiderocol } \\
\hline $\begin{array}{l}\text { Bassetti et al., } \\
2020 \text { [41] }\end{array}$ & $\begin{array}{l}\text { Phase 3, } \\
\text { randomized, } \\
\text { prospective, } \\
\text { multicentre, } \\
\text { open-label } \\
\text { (CREDIBLE- } \\
\text { CR) }\end{array}$ & $\begin{array}{l}150 \\
\text { (101 cefiderocol vs. } \\
49 \mathrm{BAT})\end{array}$ & $\begin{array}{c}\text { Cefiderocol } \\
2 \mathrm{~g} \mathrm{q} 8 \mathrm{~h} \text { (3h-infusion) } \\
100 \% \text { target therapy } \\
\\
\text { Dose adjustment } \\
\text { according to renal } \\
\text { function }\end{array}$ & $44.6 \% \mathrm{HAP} / \mathrm{VAP}$ & $15 \%$ P. aeruginosa & $\begin{array}{c}\text { ICU admission } 56 \% \\
\text { Septic shock } 19 \% \\
\text { Mechanical ventilation } \\
50 \% \\
\text { Immunocompromised } 27 \% \\
\text { Mean SOFA score } 5.1\end{array}$ & $\begin{array}{c}\text { Mortality rate in PA } \\
\text { subgroup: } \\
\text { 35\% vs. 17\% } \\
\text { ( } p=\text { NS) } \\
\text { Clinical cure at the end of } \\
\text { treatment (HAP/VAP } \\
\text { subgroup): } \\
\text { 60\% vs. 63\% }\end{array}$ & NA & $\begin{array}{l}\text { A numerically higher } \\
\text { proportion of patients } \\
\text { with CRE infections } \\
\text { achieved a clinical cure in } \\
\text { the cefiderocol group than } \\
\text { in the BAT group ( } 66 \% \text { vs. } \\
\text { 45\%). }\end{array}$ \\
\hline $\begin{array}{l}\text { Wunderink } \\
\text { et al., } 2020 \text { [42] }\end{array}$ & $\begin{array}{l}\text { Phase 3, } \\
\text { randomized, } \\
\text { prospective, } \\
\text { multicentre, } \\
\text { open-label } \\
\text { (APEKS-NP) }\end{array}$ & $\begin{array}{c}300 \\
\text { (148 cefiderocol vs. } \\
152 \text { meropenem) }\end{array}$ & $\begin{array}{c}\text { Cefiderocol } \\
2 \mathrm{~g} \text { q8h ( } 3 \mathrm{~h} \text { infusion) } \\
\text { vs. } \\
\text { MER } \\
2 \mathrm{~g} \text { q8h ( } 3 \mathrm{~h} \text { infusion })\end{array}$ & $\begin{array}{l}123 \text { VAP } \\
119 \text { HAP } \\
50 \text { HCAP }\end{array}$ & $\begin{array}{l}16.4 \% \text { P. aeruginosa } \\
8 \% \\
\text { carbapenemase- } \\
\text { producers }\end{array}$ & $\begin{array}{c}\text { ICU admission: } 68 \% \\
\text { Mechanical ventilation: } \\
60 \% \\
\text { Mean SOFA score } 4.8 \\
\text { APACHE II score } \geq 16: \\
49 \%\end{array}$ & $\begin{array}{c}\text { Mortality rate at } 14 \text {-day } \\
\text { in PA subgroup: } \\
8 \% \text { vs. } 13 \% \\
\text { ( } p=\text { NS) } \\
\text { Clinical cure rate in PA } \\
\text { subgroup: } \\
67 \% \text { vs. } 71 \% \\
\text { ( } p=\text { NS) }\end{array}$ & NA & $\begin{array}{c}\text { Cefiderocol was } \\
\text { non-inferior to high-dose, } \\
\text { extended-infusion MER in } \\
\text { terms of all-cause } \\
\text { mortality on day } 14 \text { in } \\
\text { patients with } \\
\text { Gram-negative } \\
\text { nosocomial pneumonia }\end{array}$ \\
\hline
\end{tabular}

Delgado-

Valverde et al.,
$2020[43]$

Mushtaq et al.,

2020 [44]

In vitro study

In vitro study

5 ST-175; 1 IMP+. Cefiderocol MIC range: 0.125-0.5 (100\% susceptibility) $45.5 \%$.
Kazmierczak In vitro study

321 carbapenemase-negative meropenem non-susceptible (MIC range 0.002-8; MIC $_{50} 0.12$; MIC90 1); 26

AKI: acute kidney injury; BAT: best available therapy; BSI: bloodstream infections; CI: continuous infusion; COL: colistin; CR: carbapenem-resistant; CRE: carbapenem-resistant Enterobacteriaceae; CRRT: continuous renal replacement therapy; CTT: ceftolozane-tazobactam; EI: extended infusion; HAP: hospital-acquired pneumonia; HCAP: healthcare-associated pneumonia; ICU: intensive care unit; MER: meropenem; MIC: minimum inhibitory concentration; MDR: multidrug-resistant; NA: not available; NS: not significant; OR: odds ratio; PA: Pseudomonas aeruginosa; PDR: pan drug-resistant; RRT: renal replacement therapy; SOFA: sequential organ failure assessment; VAP: ventilator-associated pneumonia; VAT ventilator-associated tracheitis; XDR: extensively drug-resistant. 
In regard to recommended dosages, the use of CI for ceftolozane-tazobactam is supported by the findings of a prospective study showing that among 72 patients affected by MDR Pseudomonas aeruginosa infections (mainly VAP), CI was associated with a higher probability of target attainment of the aggressive PK/PD target of $100 \% f \mathrm{~T}>4 \times \mathrm{MIC}$ [46]. Additionally, a case-control study evaluating 28 patients affected by MDR Pseudomonas aeruginosa infections (67.9\% pneumonia) showed that prolonging infusion up to $3 \mathrm{~h}$ allowed significantly lower resistance development compared to intermittent infusion $(0.0 \% \mathrm{vs}$. $58.3 \% ; p=0.04$ ) [47]. Consequently, we suggest CI of ceftolozane-tazobactam after loading for rapidly achieving and then maintaining aggressive PK/PD targets in IVACs caused by MDR MBL-negative GES-negative Pseudomonas aeruginosa isolates.

Continuous infusion of ceftazidime-avibactam (7.5 g/day after $2.5 \mathrm{LD})$ or cefiderocol ( $2 \mathrm{~g}$ LD followed by $2 \mathrm{~g}$ q8h in CI) in monotherapy or in association with fosfomycin (6-8 $\mathrm{g}$ LD followed by $16 \mathrm{~g}$ /day CI) represents the first-line therapy for the management of IVACs caused by MDR GES-positive Pseudomonas aeruginosa (Figure 1, Panel A.3). A summary of the evidence assessing the efficacy of ceftazidime-avibactam alone or with fosfomycin in patients affected by IVACs caused by MDR GES-positive Pseudomonas aeruginosa is provided in Table 3.

In a phase III RCT [48], Torres et al. reported no significant difference in clinical cure rate $(64.3 \%$ vs. $77.1 \% ; p=\mathrm{NS})$ between ceftazidime-avibactam and meropenem in subgroup of critically ill patients affected by HAP or VAP caused by MDR Pseudomonas aeruginosa. However, Pseudomonas aeruginosa accounted only for $30 \%$ of overall isolates, and no further analysis was provided to identify resistance mechanisms of Pseudomonas aeruginosa isolates (including carbapenemases/GES-production). Several observational studies and case series / reports [49-53] demonstrated the efficacy of ceftazidime-avibactam in critically ill patients (ICU admission ranging from $41.5 \%$ to $100 \%$ ) affected by HAP/VAP in most cases. Particularly, Vena et al. [50] reported a clinical success of $87.8 \%$ in Pseudomonas aeruginosa infections (48.8\% HAP/VAP) in 41 patients treated with ceftazidime-avibactam (prolonged infusion in $36.6 \%$ of cases). Notably, an in vitro analysis of a retrospective study including 24 patients affected by XDR Pseudomonas aeruginosa infections (33.3\% nosocomial pneumonia) found an overall susceptibility to ceftazidime-avibactam of $100 \%$ in GES-5-positive strains belonging to ST235 clone, showing a $\mathrm{MIC}_{90}$ of $6 \mathrm{mg} / \mathrm{L}$ [54].

Only preclinical evidence supported the association therapy between ceftazidimeavibactam and fosfomycin against MDR Pseudomonas aeruginosa [55-57]. Synergism between the two agents was retrieved in $25-61.9 \%$ of isolates and was also confirmed in a murine model of infection [55].

Cefiderocol could represent a valuable alternative to ceftazidime-avibactam in the treatment of IVACs caused by MBL-negative MDR GES-positive Pseudomonas aeruginosa, but caution is required due to the limited clinical experience in this setting and the quite low number of GES-positive strains that have been tested in vitro for susceptibility to this antibiotic. Anyway, considering that in most clinical studies MBL-negative MDR GESpositive Pseudomonas aeruginosa isolates were rarely characterized, caution is recommended in choosing any agent, including ceftazidime-avibactam.

In regard to recommended dosages, evidence supporting the use of CI for ceftazidimeavibactam is stemmed from a large observational study among 577 patients with KPCproducing Klebsiella pneumoniae treated with ceftazidime-avibactam, in which prolonged and/or CI was independently associated with higher survival rate compared to intermittent infusion [58]. In regard to cefiderocol, we recently showed in a descriptive case series of PK/PD target attainment and microbiological outcome in critically ill patients with documented severe XDR Acinetobacter baumannii BSI and/or VAP treated with cefidero$\mathrm{col}$ that the standard $3 \mathrm{~h}$ infusion was ineffective in achieving the aggressive PK/PD of $100 \% \mathrm{fT}>4 \times \mathrm{MIC}$ in more than $50 \%$ of included patients. This resulted in a remarkable high rate of microbiological failure, especially among VAP cases [59]. 
Table 3. Summary of the studies investigating the treatment of multidrug-resistant (MDR) metallo-beta-lactamase-negative GES-positive Pseudomonas aeruginosa infection-related ventilator-associated complications (IVACs) with ceftazidime-avibactam monotherapy or in combination with fosfomycin.

\begin{tabular}{|c|c|c|c|c|c|c|c|c|c|}
\hline $\begin{array}{l}\text { Author, Year } \\
\text { and Reference }\end{array}$ & Study Design & No. of Patients & $\begin{array}{l}\text { Antibiotic and } \\
\text { Dosing }\end{array}$ & Rate of IVACs & Isolates & Severity & $\begin{array}{l}\text { Clinical } \\
\text { Outcomes }\end{array}$ & $\begin{array}{c}\text { Relapse } \\
\text { Rate-Resistance } \\
\text { Development }\end{array}$ & Comments \\
\hline \multicolumn{10}{|c|}{ Ceftazidime-avibactam } \\
\hline $\begin{array}{l}\text { Torres et al., } \\
2018 \text { [48] }\end{array}$ & $\begin{array}{l}\text { Phase III RCT, } \\
\text { multicentre } \\
\text { (REPROVE) }\end{array}$ & $\begin{array}{c}808 \\
\text { (405 CTV } \\
\text { vs. } 403 \text { MER) }\end{array}$ & $\begin{array}{l}\text { CTV } \\
2.5 \text { g q8h } \\
\text { vs. } \\
\text { MER } \\
1 \text { g q8h }\end{array}$ & $\begin{array}{l}67 \% \text { HAP } \\
33 \% \text { VAP }\end{array}$ & $\begin{array}{c}30 \% \text { P. aeruginosa } \\
\text { CTV } \\
\mathrm{MIC}_{90}: \\
8 \mathrm{mg} / \mathrm{L}\end{array}$ & $\begin{array}{l}\text { Mechanical ventilation } \\
43 \%\end{array}$ & $\begin{array}{c}\text { Overall clinical cure: } \\
68.8 \% \text { vs. } 73 \% \\
\text { ( } p=\text { NS) } \\
\text { Clinical cure in PA } \\
\text { subgroup: } \\
64.3 \% \text { vs. } 77.1 \% \\
\text { ( } p=\mathrm{NS})\end{array}$ & NA & $\begin{array}{l}\text { CTV } \\
\text { potential alternative to } \\
\text { carbapenems in the } \\
\text { management of } \\
\text { nosocomial pneumonia, } \\
\text { also caused by PA }\end{array}$ \\
\hline $\begin{array}{l}\text { Jorgensen et al., } \\
2019 \text { [49] }\end{array}$ & $\begin{array}{l}\text { Retrospective } \\
\text { observational, } \\
\text { multicentre }\end{array}$ & 63 & $\begin{array}{c}\text { CTV } \\
2.5 \mathrm{~g} \text { q8h } \\
\text { Dose adjustment } \\
\text { according to renal } \\
\text { function }\end{array}$ & 60.3\% HAP/VAP & $\begin{array}{c}100 \% \text { P. aeruginosa } \\
\text { CTV } \\
\mathrm{MIC}_{50}: \\
2 \mathrm{mg} / \mathrm{L} \\
\mathrm{CTV} \\
\mathrm{MIC} \mathrm{C}_{90}: \\
6 \mathrm{mg} / \mathrm{L}\end{array}$ & $\begin{array}{c}\text { ICU admission } 55.6 \% \\
\text { Median SOFA score } 5 \\
\text { Immunocompromised } \\
6.3 \%\end{array}$ & $\begin{array}{c}\text { Clinical failure: } \\
30.2 \% \\
\text { 30-day mortality rate: } \\
17.5 \%\end{array}$ & $\begin{array}{l}\text { Relapse } 6.3 \% \\
\text { Resistance } 0 \%\end{array}$ & $\begin{array}{c}\text { CTV } \\
\text { could be an effective } \\
\text { therapy for MDR-PA as } \\
\text { well as CRE infections. } \\
\text { No difference in mortality } \\
\text { rate between PA and CRE } \\
\text { treated with CTV } \\
\text { (17.5\% vs. } 16.2 \% ; p=\text { NS) }\end{array}$ \\
\hline $\begin{array}{c}\text { Vena et al., } 2020 \\
\text { [50] }\end{array}$ & $\begin{array}{l}\text { Retrospective } \\
\text { observational, } \\
\text { multicentre }\end{array}$ & 41 & $\begin{array}{c}\text { CTV } \\
2.5 \mathrm{~g} \mathrm{q} 8 \mathrm{~h} \\
\text { (36.6\% CI/EI) } \\
80.5 \% \text { targeted } \\
\text { therapy } \\
\begin{array}{c}\text { Dose adjustment } \\
\text { according to renal } \\
\text { function }\end{array} \\
\end{array}$ & $\begin{array}{c}48.8 \% \text { nosocomial } \\
\text { pneumonia } \\
\text { (65\% VAP-35\% } \\
\text { HAP) }\end{array}$ & $80.5 \%$ P. aeruginosa & $\begin{array}{c}\text { ICU admission } 41.5 \% \\
\text { Mechanical ventilation } \\
34.1 \% \\
\text { Septic shock } 17.1 \% \\
\text { CRRT 12.2\% } \\
\text { Solid organ transplant } \\
\text { recipients 19.5\% } \\
\text { Haematological } \\
\text { malignancies } 9.8 \% \\
\text { Neutropenia } 12.2 \% \\
\end{array}$ & $\begin{array}{c}\text { Clinical success in } \\
\text { HAP/VAP: } \\
90 \% \\
\text { Clinical cure rate in PA } \\
\text { subgroup: } \\
87.8 \%\end{array}$ & NA & $\begin{array}{c}\text { CTV } \\
\text { as value option for } \\
\text { XDR-PA infection, } \\
\text { including HAP/VAP }\end{array}$ \\
\hline $\begin{array}{c}\text { Rodriguez- } \\
\text { Nunez, 2018 } \\
\text { [51] }\end{array}$ & Case series & 8 & $\begin{array}{c}\text { CTV } \\
2.5 \mathrm{~g} \text { q8h } \\
\text { Dose adjustment } \\
\text { according to renal } \\
\text { function }\end{array}$ & $62.5 \% \mathrm{HAP} / \mathrm{VAP}$ & MDR/XDR PA & NA & $\begin{array}{c}\text { Clinical cure rate: } \\
50 \% \\
\text { (40\% in HAP/VAP } \\
\text { subgroup) } \\
\text { 30-day mortality rate: } \\
37.5 \% \\
\text { (60\% in HAP/VAP } \\
\text { subgroup) }\end{array}$ & Relapse $20 \%$ & \\
\hline
\end{tabular}


Table 3. Cont.

\begin{tabular}{|c|c|c|c|c|c|c|c|c|c|}
\hline $\begin{array}{l}\text { Author, Year } \\
\text { and Reference }\end{array}$ & Study Design & No. of Patients & $\begin{array}{l}\text { Antibiotic and } \\
\text { Dosing }\end{array}$ & Rate of IVACs & Isolates & Severity & $\begin{array}{l}\text { Clinical } \\
\text { Outcomes }\end{array}$ & $\begin{array}{c}\text { Relapse } \\
\text { Rate-Resistance } \\
\text { Development }\end{array}$ & Comments \\
\hline $\begin{array}{l}\text { Santevecchi } \\
\text { et al., 2018 [52] }\end{array}$ & Case series & 3 & $\begin{array}{l}\text { CTV } \\
2.5 \mathrm{q} 8 \mathrm{~h} \\
\text { Dose adjustment } \\
\text { according to renal } \\
\text { function }\end{array}$ & $100 \%$ VAP & $\begin{array}{l}2 \text { MDR-PA } \\
1 \text { XDR-PA }\end{array}$ & $\begin{array}{c}\text { ICU admission } 100 \% \\
\text { Mechanical ventilation } \\
100 \%\end{array}$ & $\begin{array}{c}\text { Clinical cure rate: } \\
66.7 \%\end{array}$ & None & \\
\hline $\begin{array}{l}\text { Xipell et al., } \\
2017 \text { [53] }\end{array}$ & Case report & 1 & $\begin{array}{c}\text { CTV } \\
2.5 \mathrm{~g} \mathrm{q} 8 \mathrm{~h} \\
\end{array}$ & HAP & XDR-PA & NA & Clinical cure $100 \%$ & None & \\
\hline $\begin{array}{c}\text { Recio et al., } 2018 \\
\text { [54] }\end{array}$ & $\begin{array}{l}\text { In vitro analysis } \\
\text { of a } \\
\text { retrospective } \\
\text { study }\end{array}$ & 24 & CTV & $33.3 \% \mathrm{HAP} / \mathrm{VAP}$ & $\begin{array}{c}45.8 \% \\
\text { GES-5-positive } \\
\text { ST235 clone } \\
41.1 \% \text { VIM-2 } \\
\text { ST175 clone } \\
13.1 \% \text { non- } \\
\text { carbapenemase } \\
\text { producers }\end{array}$ & \multicolumn{4}{|c|}{$\begin{array}{l}\text { Overall susceptibility rate to CTV } \\
\text { in GES-5-positive strains:100\%; } \text { MIC }_{90} 6 \mathrm{mg} / \mathrm{L} \\
\text { CTV } \\
\text { demonstrated in vitro high activity against GES-positive strains }\end{array}$} \\
\hline
\end{tabular}

\section{Ceftazidime-Avibactam + Fosfomycin}

\begin{tabular}{|c|c|c|}
\hline $\begin{array}{c}\text { Papp } \\
\text {-Wallace et al., } \\
2019 \text { [55] }\end{array}$ & $\begin{array}{l}\text { Preclinical } \\
\text { study- } \\
\text { murine model } \\
\text { infection }\end{array}$ & $\begin{array}{l}\text { The association between CTV and FOS significantly reduced the P. aeruginosa (CFUs), by approximately } 2 \text { and } 5 \text { logs, compared with stasis and in the vehicle-treated control, respectively. Administration of } \\
\text { ceftazidime-avibactam and fosfomycin separately significantly increased CFUs, by approximately } 3 \text { logs and } 1 \text { log, respectively, compared with the number at stasis, and only reduced CFUs by } \\
\text { approximately } 1 \text { log and } 2 \text { logs, respectively, compared with the number in the vehicle-treated control. } \\
\text { The combination of CTV + FOS was superior to either drug alone and has the potential to offer infected patients with high bacterial burdens a valid therapeutic choice against infection with MDR-PA that } \\
\text { lack metallo-beta-lactamases. }\end{array}$ \\
\hline $\begin{array}{l}\text { Avery et al., } \\
2019[56]\end{array}$ & In vitro study & CR-PA: CTV baseline susceptibility $89.5 \%$. Synergism with FOS in $25 \%$ of isolates (FICI $\leq 0.5$ ) \\
\hline $\begin{array}{l}\text { Mikhail et al., } \\
2019[57]\end{array}$ & In vitro study & MDR-PA. CTV MIC reduction in 13/21 (61.9\%) of isolates in combination with FOS. Combination between CTV and FOS was indifferent at time-kill analysis. \\
\hline
\end{tabular}

CFU: colony format unit; CI: continuous infusion; CR: carbapenem-resistant; CRE: carbapenem-resistant Enterobacteriaceae; CRRT: continuous renal replacement therapy; CTV: ceftazidime-avibactam; EI: extended infusion; FICI: fractional inhibitory concentration index; FOS: fosfomycin; HAP: hospital-acquired pneumonia; ICU: intensive care unit; MER meropenem; MIC: minimum inhibitory concentration; MDR: multidrug-resistant; NA: not available; NS: not significant; PA: Pseudomonas aeruginosa; RCT; randomized controlled trial; SOFA: sequential organ failure assessment; VAP: ventilator-associated pneumonia; XDR: extensively drug-resistant. 
Consequently, we recommend CI of ceftazidime-avibactam and/or of cefiderocol after loading for rapidly achieving and then maintaining aggressive PK/PD targets in IVACs caused by MDR Pseudomonas aeruginosa, also taking into account the limited ELF penetration rate of these agents [60-62].

2.1.3. Multidrug-Resistant (MDR) Metallo-Beta-Lactamase (MBL)-Positive Pseudomonas aeruginosa

Prolonged infusion of cefiderocol ( $2 \mathrm{~g}$ LD followed by $2 \mathrm{~g}$ q8h in EI or CI) in combination therapy with inhaled colistin ( $2 \mathrm{MU} \mathrm{q} 8 \mathrm{~h}$ ) is recommended as first-line therapy for the management of IVACs caused by MDR metallo-beta-lactamase-producers (VIM, IMP, or NDM) Pseudomonas aeruginosa. Combination therapy including high-dose prolonged infusion meropenem (1-1.5 g q6h after $2 \mathrm{~g}$ LD), fosfomycin (6-8 g LD followed by $16 \mathrm{~g} /$ day $\mathrm{CI}$ ), and inhaled colistin ( $2 \mathrm{MU} \mathrm{q} 8 \mathrm{~h}$ ) should be reserved as second-line alternative (Figure 1 , panel A.4). A summary of the evidence assessing the efficacy of these antibiotic regimens in patients affected by IVACs caused by MDR metallo-beta-lactamase-positive Pseudomonas aeruginosa is provided in Table 4 .

Currently, no case of IVACs caused by metallo-beta-lactamase-positive Pseudomonas aeruginosa treated with cefiderocol exists. Real-world evidence is limited to two different case reports showing the efficacy of cefiderocol in osteomyelitis caused by NDM- or VIM-positive Pseudomonas aeruginosa [66,67]. In vitro studies $[44,45,63]$ demonstrated the activity of cefiderocol against MBL-producing Pseudomonas aeruginosa, showing an overall susceptibility respectively of $93.3 \%, 80 \%$, and $45.5 \%$ for VIM-, IMP-, or NDM-positive strains [44]. For VIM-positive isolates, MIC ranged from $0.008-0.03$ to $1-2 \mathrm{mg} / \mathrm{L}$ with a $\mathrm{MIC}_{50}$ of $0.25[45,63]$.

Although no clinical evidence for the use of meropenem in combination with fosfomycin for the management of IVACs caused by MBL-producing Pseudomonas aeruginosa currently exists, Albiero et al. [64] reported in vitro the synergic effect of the combination regimen in ten MBL-positive Pseudomonas aeruginosa. Synergism was found in $100 \%$ of isolates, resulting in a median decrease of MIC50 and MIC90 by eight-fold. PK/PD simulation showed that high-dose fosfomycin (6-8 g q8h) or meropenem (1.5 g q6h in $3 \mathrm{~h} \mathrm{EI})$ achieved the probability of target attainment of $\geq 90 \%$ respectively at an MIC of $32 \mathrm{mg} / \mathrm{L}$ and $16 \mathrm{mg} / \mathrm{L}$. Additionally, combination therapy resulted in a significantly increase in the cumulative fraction rate against MBL-positive Pseudomonas aeruginosa compared to monotherapy with meropenem (32\% vs. $68 \%$ ) or fosfomycin ( $0 \%$ vs. $74 \%$ ) [64].

A systematic review including patients affected by HAP or VAP due to MDR Pseudomonas aeruginosa reported respectively a clinical success and microbiological eradication in $70.4 \%$ and $71.3 \%$ of cases with inhaled colistin monotherapy [65]. No evidence for inhaled colistin in association with cefiderocol or meropenem/fosfomycin combination therapy currently exists. 
Table 4. Summary of the studies investigating the treatment of multidrug-resistant (MDR) metallo-beta-lactamase-positive Pseudomonas aeruginosa infection-related ventilator-associated complications (IVACs) with cefiderocol in association with inhaled colistin or combination therapy between meropenem, fosfomycin and inhaled colistin.

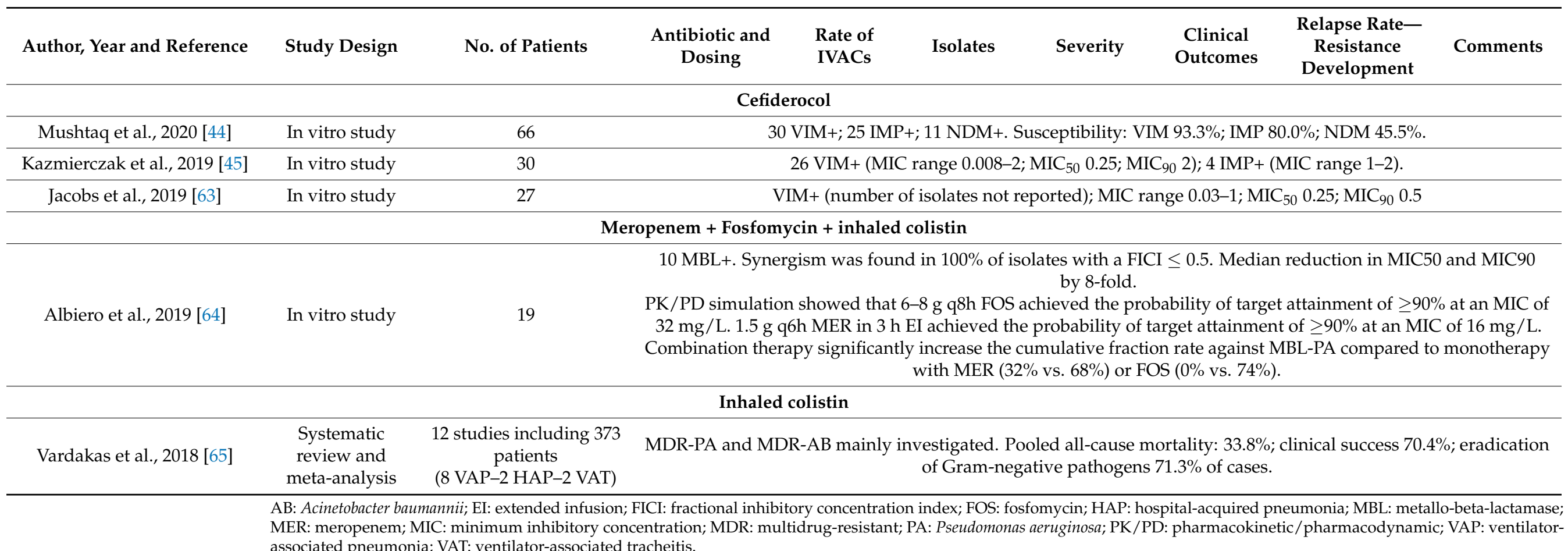

MER: meropenem; MIC: minimum inhibitory concentration; MDR: multidrug-resistant; PA: Pseudomonas aeruginosa; PK/PD: pharmacokinetic/pharmacodynamic; VAP: ventilatorassociated pneumonia; VAT: ventilator-associated tracheitis. 
In regard to recommended dosages, evidence supporting the use of high-doses CI meropenem after $2 \mathrm{~g}$ loading stemmed from a recent study that assessed the impact of maximizing Css/MIC ratio on efficacy of CI meropenem against documented Gram-negative infections in critically ill patients [68]. In that study, it was shown that a Css/MIC ratio $\geq 4.63$ was significantly associated with favourable clinical outcome among 74 ICU patients [68]. Monte Carlo simulation showed that higher meropenem dosages by CI should be recommended for dealing tackling appropriately Pseudomonas aeruginosa and Acinetobacter baumannii infections in critically ill patients with preserved renal function [68]. Likewise, a similar aggressive PK/PD target of Css/MIC > 5 within the first $72 \mathrm{~h}$ was significantly associated with both microbiological eradication and prevention of resistance development in a large cohort of critically ill patients having documented Gram-negative infections treated with CI traditional beta-lactams [22]. In regard to cefiderocol, we recently showed in a descriptive case series of PK/PD target attainment and microbiological outcome in critically ill patients with documented severe XDR Acinetobacter baumannii BSI and/or VAP that treatment with cefiderocol at the standard 3 \#h infusion was ineffective in achieving the aggressive PK/PD of $100 \% \mathrm{fT}>4 \times \mathrm{MIC}$ in more than $50 \%$ of included patients. This resulted in a remarkable high rate of microbiological failure, especially among VAP cases [59]. Consequently, we suggest CI of cefiderocol or of high dose meropenem after loading for promptly achieving and then maintaining an aggressive PK/PD target in critically ill patients affected by IVACs due to MDR Pseudomonas aeruginosa.

\subsection{Targeted Treatment of IVACs Caused by Acinetobacter baumannii in Critically Ill Adult Patients}

Therapeutic algorithm for targeted treatment of IVACs caused by Acinetobacter baumannii in adult ICU patients is shown in Figure 2.
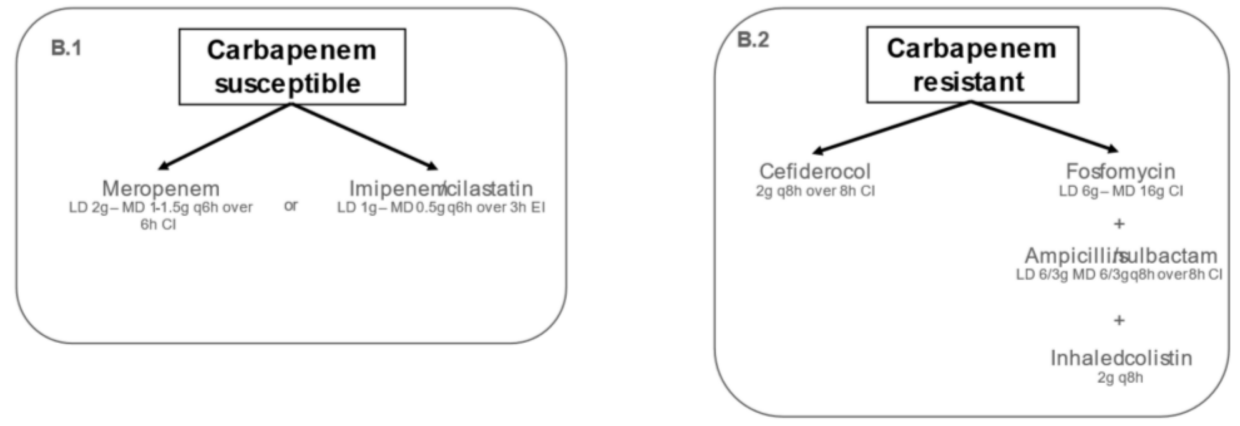

Figure 2. Algorithms for targeted treatment of IVAC caused by full-susceptible and multidrugresistant Acinetobacter baumannii. CI: continuous infusion; EI: extended infusion; LD: loading dose; MD: maintenance dose; MDR: multidrug resistance.

\subsubsection{Carbapenem-Susceptible Acinetobacter baumannii}

Continuous infusion of high-dose meropenem (1-1.5 g q6h after $2 \mathrm{~g}$ LD) or imipenem (500 mg q4-6h after $1 \mathrm{~g}$ LD) are recommended as targeted therapy for the management of IVACs caused by carbapenem-susceptible Acinetobacter baumannii (Figure 2, panel B.1). Several clinical and in vitro evidence [69-75] demonstrated the efficacy of carbapenems (particularly meropenem and imipenem) for the treatment of carbapenem-susceptible Acinetobacter baumannii infections (Table 5). 
Table 5. Summary of the studies investigating the treatment of carbapenem-susceptible Acinetobacter baumannii infection-related ventilator-associated complications (IVACs) with carbapenems.

\begin{tabular}{|c|c|c|c|c|c|c|c|c|c|}
\hline $\begin{array}{l}\text { Author, Year } \\
\text { and Reference }\end{array}$ & Study Design & No. of Patients & $\begin{array}{l}\text { Antibiotic and } \\
\text { Dosing }\end{array}$ & Rate of IVACs & Isolates & Severity & $\begin{array}{l}\text { Clinical } \\
\text { Outcomes }\end{array}$ & $\begin{array}{c}\text { Relapse } \\
\text { Rate-Resistance } \\
\text { Development }\end{array}$ & Comments \\
\hline \multicolumn{10}{|c|}{ Carbapenems (Meropenem-Imipenem) } \\
\hline $\begin{array}{c}\text { Garnacho } \\
\text {-Montero et al., } \\
2003 \text { [69] }\end{array}$ & $\begin{array}{c}\text { Prospective } \\
\text { observational }\end{array}$ & $\begin{array}{c}35 \\
\text { (21 colistin } \\
\text { vs. } \\
14 \text { imipenem) }\end{array}$ & $\begin{array}{l}\text { Colistin } \\
2.5-5 \mathrm{mg} / \mathrm{kg} / \text { day in } \\
\text { three doses } \\
\text { vs. } \\
\text { Impenem } \\
2-3 \mathrm{~g} / \text { day in } \\
\text { three } / \text { four } \\
\text { doses }\end{array}$ & $100 \%$ VAP & $\begin{array}{c}21 \\
\text { carbapenem } \\
\text {-resistant AB } \\
14 \\
\text { carbapenem- } \\
\text { susceptible AB }\end{array}$ & $\begin{array}{c}\text { ICU admission } 100 \% \\
\text { Septic shock } 57.1 \% \\
\text { APACHE II score: } \\
20.5 \pm 7 \\
\text { SOFA score: } 11.7 \pm 6.6\end{array}$ & $\begin{array}{c}\text { Clinical cure rate: } \\
57 \% \text { vs. } 57 \% \\
\text { ( } p=\text { NS) } \\
\text { Mortality rate: } \\
61.9 \% \text { vs. } 64.2 \% \\
\text { ( } p=\text { NS) } \\
\text { VAP-related mortality } \\
\text { rate: } \\
38.0 \% \text { vs. } 35.7 \% \\
(p=\text { NS })\end{array}$ & NA & $\begin{array}{l}\text { No difference in efficacy } \\
\text { and safety between } \\
\text { carbapenem and } \\
\text { intravenous colistin in } \\
\text { the management of VAP } \\
\text { caused by MDR-AB }\end{array}$ \\
\hline $\begin{array}{l}\text { Kallel et al., } \\
2007 \text { [70] }\end{array}$ & $\begin{array}{l}\text { Retrospective } \\
\text { matched } \\
\text { case-control }\end{array}$ & $\begin{array}{c}120 \\
(60 \text { colistin vs. } \\
60 \text { imipenem })\end{array}$ & $\begin{array}{c}\text { Colistin } \\
2 \text { MU q8h } \\
\text { vs. } \\
\text { Imipenem } \\
500 \mathrm{mg} \mathrm{q6h}\end{array}$ & $100 \% \mathrm{VAP}$ & $\begin{array}{c}61.7 \% \\
\text { carbapenem- } \\
\text { susceptible AB } \\
38.3 \% \\
\text { carbapenem- } \\
\text { susceptible } P . \\
\text { aeruginosa } \\
\text { (in patients } \\
\text { receiving imipenem) }\end{array}$ & $\begin{array}{l}\text { ICU admission } 100 \% \\
\text { SAPS-II } 33.2 \pm 10.8 \\
\text { Septic shock } 23.3 \%\end{array}$ & $\begin{array}{l}\text { Clinical cure rate: } \\
75 \% \text { vs. } 71.7 \% \\
\quad(p=0.68) \\
\\
\text { Mortality rate: } \\
41.7 \% \text { vs. } 35 \% \\
\quad(p=0.45)\end{array}$ & $\begin{array}{c}\text { Relapse: } \\
8.3 \% \\
\text { Resistance } \\
\text { development: } \\
0.0 \%\end{array}$ & $\begin{array}{l}\text { No difference in efficacy } \\
\text { and safety between } \\
\text { carbapenem and } \\
\text { intravenous colistin in } \\
\text { the management of VAP } \\
\text { caused by MDR-AB }\end{array}$ \\
\hline $\begin{array}{l}\text { Ikonomidis } \\
\text { et al., 2006 [72] }\end{array}$ & In vitro study & 320 & \multicolumn{7}{|c|}{$40.6 \%$ resistance to meropenem $\left(\mathrm{MIC}_{50} 4 \mathrm{mg} / \mathrm{L} ; \mathrm{MIC}_{90} 8 \mathrm{mg} / \mathrm{L}\right) ; 67.8 \%$ resistance to imipenem ( $\mathrm{MIC}_{50} 8 \mathrm{mg} / \mathrm{L} \mathrm{MIC} \mathrm{C}_{90} 64 \mathrm{mg} / \mathrm{L}$ ) } \\
\hline $\begin{array}{l}\text { Mezzatesta } \\
\text { et al., 2008 [73] }\end{array}$ & In vitro study & 107 & \multicolumn{7}{|c|}{ 88.8\% MDR-AB. 59\% resistance to meropenem (MIC $906 \mathrm{mg} / \mathrm{L}$ ); $50 \%$ resistance to imipenem ( $\mathrm{MIC}_{90} 32 \mathrm{mg} / \mathrm{L}$ ) } \\
\hline $\begin{array}{c}\text { Guzek et al., } \\
2013 \text { [74] }\end{array}$ & In vitro study & 54 & \multicolumn{7}{|c|}{$22.2 \%$ resistance to doripenem; $22.2 \%$ resistance to imipenem; $42.6 \%$ resistance to meropenem } \\
\hline $\begin{array}{l}\text { Jones et al., } 2005 \\
{[75]}\end{array}$ & In vitro study & 33 & \multicolumn{7}{|c|}{$\begin{array}{c}100 \% \text { wild-type Acinetobacter spp isolates. } 75.8 \% \text { susceptibility to meropenem }\left(\text { MIC }_{90}>8 \mathrm{mg} / \mathrm{L} \text { ); } 75.8 \% \text { susceptibility to imipenem (MIC } \mathrm{C}_{90}>8 \mathrm{mg} / \mathrm{L} \text { ); } 75.8 \% \text { susceptibility to }\right. \\
\text { doripenem }\left(\mathrm{MIC}_{90} 16 \mathrm{mg} / \mathrm{L} \text { ) }\right.\end{array}$} \\
\hline
\end{tabular}


Two different observational studies $[69,70]$ compared imipenem with intravenous colistin for the management of critically ill patients affected by VAP due to MDR Acinetobacter baumannii, reporting no significant difference in clinical cure rate, mortality rate, resistance development, and adverse events between the two agents. Wang [71] retrospectively evaluated 30 critically ill patients affected by HAP/VAP due to MDR carbapenem-susceptible Acinetobacter baumannii, reporting a clinical cure rate of $100.0 \%$. Furthermore, the administration of meropenem in EI was a cost-effective approach in this setting, although showing an equal clinical efficacy compared to intermittent infusion. Several in vitro studies [72-75] reported a variable susceptibility to carbapenems of Acinetobacter baumannii strains in different ecological settings, ranging from $41 \%$ to $75.8 \%$ and from $32.2 \%$ to $77.8 \%$ for meropenem and imipenem, respectively.

In regard to recommended dosages, evidence supporting the use of high-doses CI meropenem after $2 \mathrm{~g}$ LD stemmed from a PK/PD analysis carried out among 74 ICU patients affected by documented Gram-negative infections (of which pneumonia accounted for half of cases), in which a Css/MIC ratio $\geq 4.63$ was significantly associated with favourable clinical outcome [68]. Monte Carlo simulation showed that, according to cumulative fraction of response, higher meropenem dosages by CI should be recommended for the management of Acinetobacter baumannii related infections among patients with preserved renal function [68]. Likewise, a similar aggressive PK/PD target of Css/MIC > 5 within the first $72 \mathrm{~h}$ was significantly associated with both microbiological eradication and prevention of resistance development in a large cohort of critically ill patients having documented Gram-negative infections treated with CI traditional beta-lactams [22].

\subsubsection{Carbapenem-Resistant Acinetobacter baumannii}

Prolonged infusion of cefiderocol ( $2 \mathrm{~g}$ q8h EI or CI after $2 \mathrm{~g}$ LD) represents the first-line therapy in the management of IVACs caused by MDR Acinetobacter baumannii. Combination therapy including fosfomycin (6-8 g LD followed by 16-24 g/day CI), high-dose ampicillinsulbactam (6 g/3g q8h CI after 6-8 g/3-4 g LD), and inhaled colistin (2 MU q8h) could be considered as second-line therapeutic alternative (Figure 2, panel B.2). A summary of the evidence assessing the efficacy of these antibiotic regimens in patients affected by IVACs caused by MDR Acinetobacter baumannii is provided in Table 6. 
Table 6. Summary of the studies investigating the treatment of carbapenem-resistant Acinetobacter baumannii infection-related ventilator-associated complications (IVACs) with cefiderocol or combination therapy between fosfomycin and ampicillin/sulbactam.

\begin{tabular}{|c|c|c|c|c|c|c|c|c|c|}
\hline $\begin{array}{l}\text { Author, } \\
\text { Year and } \\
\text { Reference }\end{array}$ & Study Design & No. of Patients & $\begin{array}{l}\text { Antibiotic and } \\
\text { Dosing }\end{array}$ & $\begin{array}{l}\text { Rate of } \\
\text { IVACs }\end{array}$ & Isolates & Severity & $\begin{array}{l}\text { Clinical } \\
\text { Outcomes }\end{array}$ & $\begin{array}{c}\text { Relapse } \\
\text { Rate- } \\
\text { Resistance } \\
\text { Development }\end{array}$ & Comments \\
\hline \multicolumn{10}{|c|}{ Cefiderocol } \\
\hline $\begin{array}{c}\text { Bassetti } \\
\text { et al., 2020 } \\
\text { [41] }\end{array}$ & $\begin{array}{l}\text { Phase 3, } \\
\text { randomized, } \\
\text { prospective, } \\
\text { multicentre, } \\
\text { open-label } \\
\text { (CREDIBLE- } \\
\text { CR) }\end{array}$ & $\begin{array}{c}150 \\
(101 \text { cefiderocol } \\
\text { vs. } 49 \text { BAT })\end{array}$ & $\begin{array}{l}\text { Cefiderocol } \\
2 \text { g q8h } \\
\text { (3h-infusion) } \\
100 \% \text { target } \\
\text { therapy } \\
\text { Dose } \\
\text { adjustment } \\
\text { according to } \\
\text { renal function }\end{array}$ & $\begin{array}{c}44.6 \% \\
\text { HAP/VAP }\end{array}$ & $\begin{array}{c}65 \% A . \\
\text { baumannii }\end{array}$ & $\begin{array}{c}\text { ICU admission } 56 \% \\
\text { Septic shock } 19 \% \\
\text { Mechanical } \\
\text { ventilation } 50 \% \\
\text { Immunocompromised } \\
27 \% \\
\text { Mean SOFA score } 5.1\end{array}$ & $\begin{array}{c}\text { Mortality rate in } \mathrm{AB} \\
\text { subgroup: } \\
49 \% \text { vs. } 18 \% \\
\text { ( } p=0.04) \\
\\
\text { Clinical cure at the } \\
\text { end of treatment } \\
\text { (HAP/VAP } \\
\text { subgroup) } \\
: 60 \% \text { vs. } 63 \% \\
\end{array}$ & NA & $\begin{array}{l}\text { A significant higher } \\
\text { mortality rate in } \\
\text { patients affected by } \\
\text { AB infections was } \\
\text { found with } \\
\text { cefiderocol } \\
\text { compared to BAT }\end{array}$ \\
\hline $\begin{array}{l}\text { Gatti et al., } \\
2021 \text { [59] }\end{array}$ & Case series & 13 & $\begin{array}{l}\text { Cefiderocol } \\
1.5-2 \mathrm{~g} \mathrm{q} 8 \mathrm{~h}\end{array}$ & $84.6 \%$ & $100 \%$ XDR-AB & $\begin{array}{c}100 \% \text { ICU } \\
\text { admission } \\
100 \% \text { mechanical } \\
\text { ventilation }\end{array}$ & $\begin{array}{c}\text { 30-day mortality } \\
\text { rate: } \\
30.8 \% \\
\\
\text { Microbiological } \\
\text { failure: } \\
54 \%\end{array}$ & NA & $\begin{array}{c}\text { Microbiological } \\
\text { failure occurred in } \\
80 \% \text { of patients with } \\
\text { suboptimal } \\
f C_{\text {min }} / \text { MIC } \\
\text { compared to } 29 \% \text { of } \\
\text { those achieving } \\
\text { optimal or } \\
\text { quasi-optimal } \\
f C_{\text {min }} / \text { MIC ratio. }\end{array}$ \\
\hline $\begin{array}{c}\text { Bavaro et al., } \\
2021 \text { [76] }\end{array}$ & Case series & 13 & $\begin{array}{l}\text { Cefiderocol } \\
2 \text { g q8h } \\
\text { (3h-infusion) } \\
\text { Dose } \\
\text { adjustment } \\
\text { according to } \\
\text { renal function }\end{array}$ & $7.7 \%$ & $\begin{array}{c}76.9 \% \\
\text { Carbapenem- } \\
\text { resistant-AB } \\
15.4 \% \text { XDR-PA } \\
7.7 \% \text { KPC }\end{array}$ & $38.5 \%$ ICU admission & $\begin{array}{c}\text { 30-day mortality } \\
\text { rate: } \\
23.1 \% \\
\\
\text { Microbiological } \\
\text { eradication: } \\
100.0 \%\end{array}$ & NA & $\begin{array}{l}\text { Combination therapy } \\
\text { with fosfomycin was } \\
\text { successfully } \\
\text { implemented in } 9 \\
\text { cases, including VAP } \\
\text { due to carbapenem- } \\
\text { resistant } \\
\text { AB }\end{array}$ \\
\hline
\end{tabular}


Table 6. Cont.

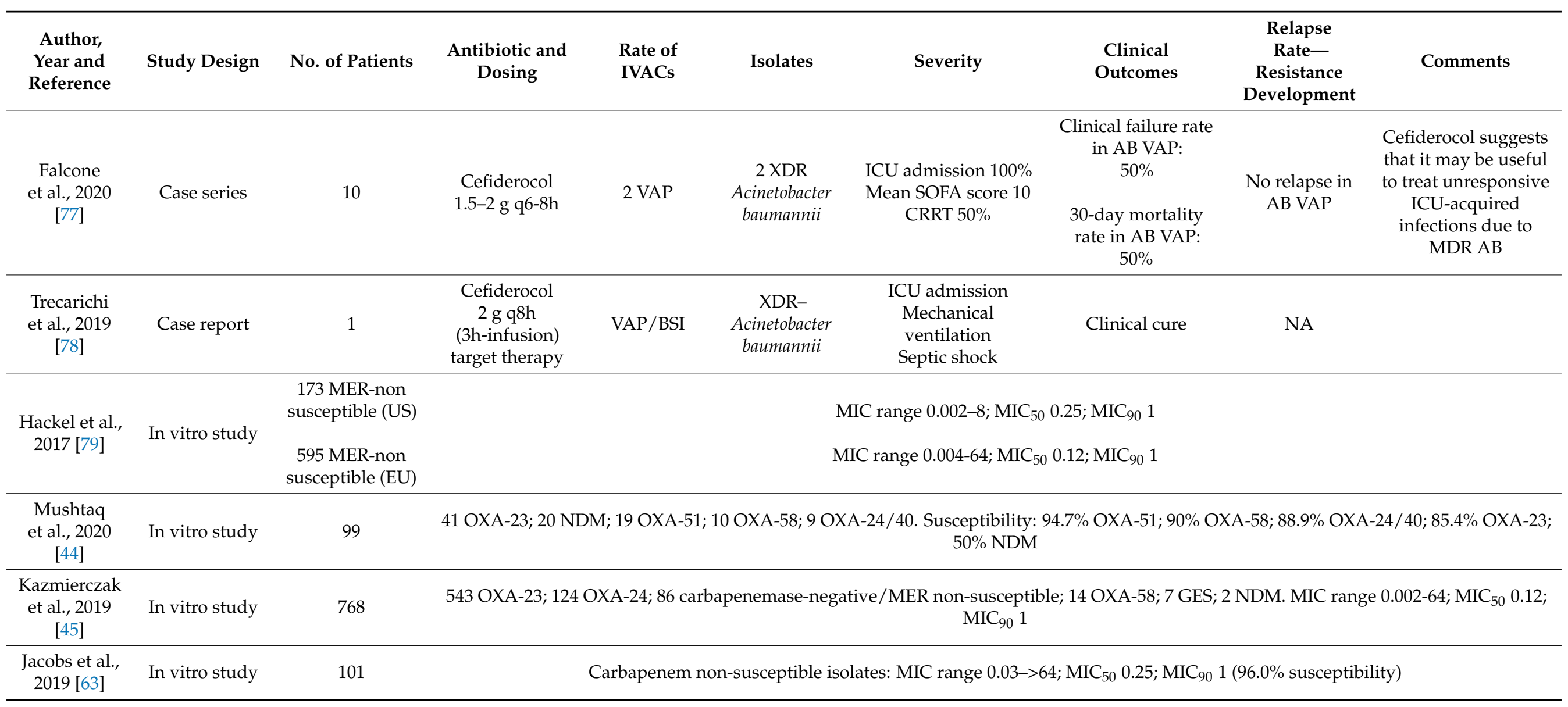


Table 6. Cont.

\begin{tabular}{|c|c|c|c|c|c|c|c|c|c|}
\hline $\begin{array}{l}\text { Author, } \\
\text { Year and } \\
\text { Reference }\end{array}$ & Study Design & No. of Patients & $\begin{array}{l}\text { Antibiotic and } \\
\text { Dosing }\end{array}$ & $\begin{array}{l}\text { Rate of } \\
\text { IVACs }\end{array}$ & Isolates & Severity & $\begin{array}{l}\text { Clinical } \\
\text { Outcomes }\end{array}$ & $\begin{array}{c}\text { Relapse } \\
\text { Rate- } \\
\text { Resistance } \\
\text { Development }\end{array}$ & Comments \\
\hline \multicolumn{10}{|c|}{ Fosfomycin + Ampicillin/Sulbactam } \\
\hline $\begin{array}{c}\text { Betrosian } \\
\text { et al., } 2007 \\
{[80]}\end{array}$ & $\mathrm{RCT}$ & 27 & $\begin{array}{c}\text { AMS } \\
6 \mathrm{~g} / 3 \mathrm{~g} \text { q } 8 \mathrm{~h} \\
\text { vs. } \\
\text { AMS } \\
8 \mathrm{~g} / 4 \mathrm{~g} \mathrm{q} 8 \mathrm{~h}\end{array}$ & $100 \% \mathrm{VAP}$ & $\begin{array}{c}\text { MDR } \\
\text { Acinetobacter } \\
\text { baumannii }\end{array}$ & $\begin{array}{l}\text { ICU admission 100\% } \\
\text { Mechanical } \\
\text { ventilation } 100 \% \\
\text { Mean APACHE II } \\
\text { score } 15\end{array}$ & $\begin{array}{l}\text { Clinical cure rate: } \\
64.3 \% \text { vs. } 69.2 \% \\
\quad(p=0.79) \\
\text { 30-day } \\
\text { mortality rate: } \\
42.9 \% \text { vs. } 53.8 \% \\
(p=N S)\end{array}$ & NA & $\begin{array}{l}\text { The use of high-dose } \\
\text { AMS regimens is } \\
\text { effective for the } \\
\text { treatment of VAP } \\
\text { caused by MDR-AB. }\end{array}$ \\
\hline $\begin{array}{c}\text { Betrosian } \\
\text { et al., 2008 } \\
{[81]}\end{array}$ & $\begin{array}{l}\text { Prospective } \\
\text { observational }\end{array}$ & $\begin{array}{c}28 \\
\text { (15 COL vs. } 13 \\
\text { AMS) }\end{array}$ & $\begin{array}{c}\text { AMS } \\
6 \mathrm{~g} / 3 \mathrm{~g} \text { q } 8 \mathrm{~h} \\
\text { vs. } \\
\text { COL } \\
3 \mathrm{MU} \text { q } 8 \mathrm{~h}\end{array}$ & $100 \% \mathrm{VAP}$ & $\begin{array}{c}\text { MDR } \\
\text { Acinetobacter } \\
\text { baumannii }\end{array}$ & $\begin{array}{c}\text { ICU admission } 100 \% \\
\text { Mechanical } \\
\text { ventilation } 100 \% \\
\text { Mean APACHE II } \\
\text { score } 14\end{array}$ & $\begin{array}{c}\text { Clinical cure rate: } \\
61.5 \% \text { vs. } 60 \% \\
\text { ( } p=\mathrm{NS}) \\
\\
28-\text { day } \\
\text { mortality rate: } \\
30 \% \text { vs. } 33 \% \\
(p=\mathrm{NS})\end{array}$ & NA & $\begin{array}{l}\text { COL and high-dose } \\
\text { AMS were } \\
\text { comparably safe and } \\
\text { effective treatments } \\
\text { for critically ill } \\
\text { patients with MDR } \\
\text { A. baumannii VAP. }\end{array}$ \\
\hline $\begin{array}{l}\text { Mellon et al., } \\
2012 \text { [82] }\end{array}$ & Case report & 1 & $\begin{array}{c}\text { AMS } \\
3 \mathrm{~g} / 1.5 \mathrm{~g} \mathrm{q} 4 \mathrm{~h} \\
+ \\
\text { FOS } \\
4 \mathrm{~g} \mathrm{q} 6 \mathrm{~h}\end{array}$ & Meningitis & $\begin{array}{c}\text { MDR } \\
\text { Acinetobacter } \\
\text { baumannii } \\
\text { MIC AMS } \\
32 \mathrm{mg} / \mathrm{L}\end{array}$ & ICU admission & Clinical cure & NA & $\begin{array}{l}\text { The only case } \\
\text { reporting the clinical } \\
\text { efficacy of } \\
\text { combination therapy } \\
\text { between fosfomycin } \\
\text { and high-dose } \\
\text { sulbactam for the } \\
\text { management of } \\
\text { deep-seated AB } \\
\text { infection. }\end{array}$ \\
\hline
\end{tabular}


Table 6. Cont.

\begin{tabular}{|c|c|c|c|c|c|c|c|c|c|}
\hline $\begin{array}{l}\text { Author, } \\
\text { Year and } \\
\text { Reference }\end{array}$ & Study Design & No. of Patients & $\begin{array}{l}\text { Antibiotic and } \\
\text { Dosing }\end{array}$ & $\begin{array}{l}\text { Rate of } \\
\text { IVACs }\end{array}$ & Isolates & Severity & $\begin{array}{l}\text { Clinical } \\
\text { Outcomes }\end{array}$ & $\begin{array}{c}\text { Relapse } \\
\text { Rate- } \\
\text { Resistance } \\
\text { Development }\end{array}$ & Comments \\
\hline $\begin{array}{l}\text { Mohd Sazlly } \\
\text { Lim et al., } \\
2021 \text { [83] }\end{array}$ & In vitro study & 50 & \multicolumn{7}{|c|}{$\begin{array}{l}\text { Fosfomycin in combination with sulbactam showed synergism in } 74 \% \text { of } \mathrm{AB} \text { isolates, resulting in a median } \mathrm{MIC}_{50} \text { and } \mathrm{MIC}_{90} \text { reduction } \\
\text { respectively of } 4-8 \text {-fold. }\end{array}$} \\
\hline \multicolumn{10}{|c|}{ Inhaled colistin } \\
\hline $\begin{array}{c}\text { Vardakas } \\
\text { et al., 2018 } \\
{[65]}\end{array}$ & $\begin{array}{l}\text { Systematic } \\
\text { review and } \\
\text { meta-analysis }\end{array}$ & $\begin{array}{c}12 \text { studies } \\
\text { including } 373 \\
\text { patients } \\
(8 \mathrm{VAP}-2 \\
\text { HAP-2 VAT) }\end{array}$ & \multicolumn{7}{|c|}{$\begin{array}{l}\text { MDR-PA and MDR-AB mainly investigated. } \\
\text { Pooled all-cause mortality: } 33.8 \% \text {; clinical success } 70.4 \% \text {; eradication of Gram-negative pathogens } 71.3 \% \text { of cases. }\end{array}$} \\
\hline $\begin{array}{l}\text { Kuo et al., } \\
2012[84]\end{array}$ & $\begin{array}{l}\text { Retrospective, } \\
\text { case-control }\end{array}$ & $\begin{array}{c}78 \\
(39 \\
\text { inhaled colistin } \\
\text { + other } \\
\text { antibiotics with } \\
\text { activity against } \\
\text { AB vs. 39 other } \\
\text { antibiotics with } \\
\text { activity against } \\
\text { AB) }\end{array}$ & Inhaled COL & $\begin{array}{c}41 \% \\
\text { HAP/VAP } \\
59 \% \\
\text { respiratory } \\
\text { colonization }\end{array}$ & $\begin{array}{c}100 \% \\
\text { MDR-AB }\end{array}$ & $\begin{array}{c}\text { ICU admission } 71.8 \% \\
\text { Mechanical } \\
\text { ventilation } 38.5 \% \\
\text { RRT } 7.7 \% \\
\text { APACHE II score } \\
20.0 \pm 6.2\end{array}$ & $\begin{array}{c}\text { Microbiological } \\
\text { eradication at } \\
\text { 14-day: } \\
84.6 \% \text { vs. } 10.3 \% \\
\text { ( } p<0.001) \\
\\
\text { 28-day mortality } \\
\text { rate: } \\
12.8 \% \text { vs. } 10.3 \% \\
(p=0.72)\end{array}$ & $\begin{array}{c}\text { Relapse rate: } \\
21.2 \% \\
\text { COL MIC } \\
\text { increase: } \\
28.6 \%\end{array}$ & $\begin{array}{l}\text { The use of inhaled } \\
\text { COL was the only } \\
\text { independent factor } \\
\text { associated with the } \\
\text { eradication of } \\
\text { MDR-AB within } \\
14 \text { days after the } \\
\text { index day (OR } \\
266.33 ; 95 \% \mathrm{CI} \\
11.26-6302.18, \\
p<0.001), \text { and } \\
\text { shortened the } \\
\text { duration of MDR-AB } \\
\text { recovery from the } \\
\text { respiratory tract by } \\
13.3 \pm 1.45 \text { days. }\end{array}$ \\
\hline
\end{tabular}


Although in vitro studies $[44,45,63,79]$ reported high susceptibility rate of carbapenem non-susceptible Acinetobacter baumannii isolates against cefiderocol ( $\mathrm{MIC}_{50}$ ranging from $0.12 \mathrm{mg} / \mathrm{L}$ to $0.25 \mathrm{mg} / \mathrm{L}$, and susceptibility above the $85 \%$ in all different OXA-producing strains), clinical evidence are currently limited. In the CREDIBLE-CR RCT [41], a significant higher mortality rate in IVAC s caused by Acinetobacter baumannii (accounting for 65\% of $\mathrm{HAP} / \mathrm{VAP}$ ) was reported in patients treated with cefiderocol compared to best available therapy ( $49 \%$ vs. $18 \% ; p=0.04)$. In a case series including 13 patients, Gatti et al. [59] found a microbiological failure rate of $54 \%$ in critically ill patients affected by XDR Acinetobacter baumannii infections (84.6\% VAP). Notably, microbiological failure occurred in $80 \%$ of patients with suboptimal $f \mathrm{C}_{\min } / \mathrm{MIC}<1$ compared to $29 \%$ of those achieving optimal $\left(f \mathrm{C}_{\min } / \mathrm{MIC}>4\right)$ or quasi-optimal $\left(f \mathrm{C}_{\min } / \mathrm{MIC}=1-4\right) \mathrm{PK} / \mathrm{PD}$ target. In a case series, Falcone et al. [77] described two critically ill COVID-19 patients developing VAP caused by XDR Acinetobacter baumannii and treated with cefiderocol. Clinical failure was reported in 50\% of cases. Conversely, Trecarichi et al. [78] reported a successful case of bacteraemic VAP caused by XDR Acinetobacter baumannii treated with cefiderocol as targeted therapy.

Different RCTs and observational studies $[80,81,83]$ demonstrated the efficacy of highdose sulbactam (up to $12 \mathrm{~g}$ /day) in monotherapy or combination therapy in IVACs caused by MDR Acinetobacter baumannii, showing no difference in clinical cure rate compared to colistin-based regimens [81]. However, evidence supporting combination therapy with fosfomycin are currently scanty. A case report [82] demonstrated the efficacy of combination therapy between high-dose sulbactam (9 g/day) and fosfomycin (16 g/day) in a critical care patients affected by meningitis due to MDR Acinetobacter baumannii. Notably, a synergism between sulbactam and fosfomycin was reported in 74\% of MDR Acinetobacter baumannii isolates, resulting in a median $\mathrm{MIC}_{50}$ and $\mathrm{MIC}_{90}$ decrease up to eight-fold [83].

A systematic review including patients affected by HAP or VAP due to MDR Acinetobacter baumannii reported respectively a clinical success and microbiological eradication in $70.4 \%$ and $71.3 \%$ of cases with inhaled colistin monotherapy [65]. A retrospective casecontrol study [84] assessing 78 patients affected by respiratory infection or colonization due to MDR Acinetobacter baumannii found that the use of inhaled colistin was the only independent factor associated with microbiological eradication within 14 days after the index day (OR 266.33; 95\% CI 11.26-6302.18, $p<0.001$ ), and shortened the duration of MDR Acinetobacter baumannii recovery from the respiratory tract by 13.3 days.

In regard to recommended dosages, no evidence for administering cefiderocol by $\mathrm{CI}$ still exists Currently. However, it was shown in a descriptive case series of PK/PD target attainment and microbiological outcome in critically ill patients with documented severe XDR Acinetobacter baumannii BSI and/or VAP that treatment with cefiderocol with the standard $3 \mathrm{~h}$ infusion was ineffective in achieving the aggressive PK/PD of $100 \% \mathrm{fT}>4 \times \mathrm{MIC}$ in more than $50 \%$ of included patients. This resulted in a remarkable high rate of microbiological failure, especially among VAP cases [59].

Consequently, we recommend CI of cefiderocol after loading for rapidly achieving and then maintaining aggressive PK/PD targets in IVACs caused by Acinetobacter baumannii, also taking into account the limited ELF penetration rate of these agents [60-62].

\section{Overview of Recommendations}

Non-fermenting Gram-negative pathogens represent a leading cause of HAP or VAP in critically ill patients requiring mechanical ventilation [2]. Particularly, Pseudomonas aeruginosa represents one of the leading causative pathogens in critically ill patients affected by HAP or VAP [85]. The widespread diffusion of MDR and XDR isolates coupled with the emergence of high-risk clones (ST111, ST175, and ST235) calls for the prompt administration of adequate antibiotic therapy and optimization of lung exposure [23,85].

Two main cornerstones should be pursued in the management of IVACs caused by Pseudomonas aeruginosa: (1) the implementation of carbapenem-sparing regimens both in case of multi-susceptible isolates (considering the higher risk of relapse or resistance development $[16,18])$, preferring the use of piperacillin-tazobactam or third/fourth-generation 
cephalosporins (ceftazidime or cefepime), and of MDR/XDR isolates, favouring the administration of novel BL/BLIs (ceftolozane-tazobactam or ceftazidime-avibactam) or cefiderocol, according to the resistance mechanism exhibited by the specific strain); (2) the adoption of altered dosing strategies (high-doses and/or prolonged infusion) in order to achieve optimal PK/PD target at the site of infection, considering the limited pulmonary penetration of piperacillin-tazobactam, ceftazidime, cefepime, ceftazidime-avibactam, and cefiderocol (below 20\%) [60-62,86-88]. Notably, in a prospective observational study including 72 patients affected by MDR Pseudomonas aeruginosa infections (79\% ICU admission; $66.7 \%$ HAP/VAP), Plimis et al. [46] reported that intermittent infusion of ceftolozane-tazobactam was inadequate to achieve optimal PK/PD target for MIC $\geq 4 \mathrm{mg} / \mathrm{L}$ compared to continuous infusion. Similarly, ATS/IDSA guidelines recommended the use of prolonged infusion of piperacillin-tazobactam, ceftazidime, or cefepime in HAP or VAP due to Pseudomonas aeruginosa to maximize lung exposure [15].

The treatment of IVACs caused by MBL-producing Pseudomonas aeruginosa is challenging. In this scenario, cefiderocol could play a major role, although evidence is currently limited to only in vitro studies. The administration of inhaled colistin could represent a valuable therapeutic strategy in association with cefiderocol or meropenem and fosfomycin, providing a targeted antibiotic delivery in respiratory tract infections and resulting in lower systemic toxicity compared to intravenous colistin [89,90]. It should not be overlooked that after administration of a single $2 \mathrm{MU}$ of inhaled colistimethate, colistin concentrations in the ELF ranged between 9.53 and $1137 \mathrm{mg} / \mathrm{L}$, which are values much higher than that achievable after the administration of the same dose by the intravenous route $(1.48-28.9 \mathrm{mg} / \mathrm{L})$ [91]. The best dosing regimen of inhaled colistimethate has still to be defined, as quite variable dosages have been proposed in the literature, ranging from $1 \mathrm{MU}$ q12h up to $5 \mathrm{MU}$ q8h [91]. However, we believe that the dosage of $2 \mathrm{MU}$ q8h should be preferred Currently considering that is the one supported by the major clinical evidence $[65,91]$.

MDR or XDR Acinetobacter baumannii is a leading causative pathogen in critically ill patients affected by VAP, characterized by mortality rate up to $60 \%$ [92]. Although colistinor polymyxin-based regimens were widely used in clinical practice for the management of severe Acinetobacter baumannii infections, relevant toxicity (mainly nephrotoxicity) and $\mathrm{PK} / \mathrm{PD}$ issues (low ELF exposure, high occurrence of breakthrough infections) strongly affect their efficacy [92].

Notably, CREDIBLE-CR trial found [41] a remarkable mortality rate amongst patients affected by Acinetobacter baumannii infections, thus mitigating the initial expectations for cefiderocol [93]. Recently, it has been suggested to limit the use of cefiderocol to situations when intolerance or resistance to other generally active drugs has been shown [93]. However, the favourable safety profile, the high in vitro activity, and the potential maximization of lung exposure through the implementation of CI, make cefiderocol the first-line choice for targeted therapy of IVACs due to MDR Acinetobacter baumannii in critically ill patients.

By virtue of their lung penetration [94], fosfomycin could be a valid alternative for combination therapy with high-dose sulbactam (9-12 g/day) as second-line strategy for the management of MDR Acinetobacter baumannii. A recent observational study [95] identified fosfomycin-containing regimen as an independent predictor for 30-day survival in severe pneumonia caused by MDR Acinetobacter baumannii. However, none of these combination therapies included sulbactam.

Overall, in the management of IVACs caused by non-fermenting Gram-negative pathogens, the implementation of beta-lactams altered dosing strategies consisting in high-dose $\mathrm{CI}$ administration is strongly recommended for attaining aggressive PK/PD target of $100 \%$ fT $>4-8 \times$ MIC and maximizing lung exposure $[87,96,97]$. This approach may both maximize clinical efficacy and prevent the development of resistance $[87,98]$. This aggressive strategy should be pursued also in the treatment of infections caused by wild-type strains. Optimal exposure into the ELF is difficult to be achieved and maintained with intermittent infusion of piperacillin-tazobactam, ceftazidime, and/or cefepime due 
to the limited penetration rates of these hydrophilic agents, and conversely, Pseudomonas aeruginosa was found to be an independent predictor of microbiological failure in critically ill patients affected by documented Gram-negative infections [22]. Clinicians must be aware that the antibiotic dosing regimens that we recommended throughout the manuscript are focused only on treatment of patients with normal renal function. It should not be overlooked that the pharmacokinetics of hydrophilic antimicrobial agents, namely beta-lactams and fosfomycin, may be affected among critically ill patients by several pathophysiological conditions that may alter volume of distribution and/or renal clearance [97,99]. Consequently, dose adjustments are needed in critically ill renal patients, especially among those with transient acute kidney injury, augmented renal clearance (ARC), and/or undergoing renal replacement therapy (RRT) [97,100]. In this scenario, adaptative daily therapeutic drug monitoring (TDM) may represent a valuable tool in addressing these issues. Currently, routinely TDM of BLs is strongly recommended for optimizing treatment among critically ill patients with Gram-negative infections [101], and an aggressive PK/PD target is considered useful among patients undergoing CRRT or having ARC [97]. Interestingly, the achievement of aggressive PK/PD target of Css/MIC ratio around 5 within the first $72 \mathrm{~h}$ of treatment with CI traditional beta-lactams was recently shown to be associated with significantly higher probability of both clinical outcome and microbiological eradication among critically ill patients with documented Gram-negative infections [22,68]. This suggests that early optimization of drug exposure by means of real-time TDM may be extremely helpful in maximizing treatment with beta lactams among the critically ill patients. However, it should be recognized that the extensive use of a real-time TDM-guided clinical pharmacologist advice approach is still burdened by many barriers [97,102-104]. To mention some of these, measurement of unbound concentrations, daily TDM assessment, timely turnaround time, appropriate interpretation of TDM data performed by well-trained MD or PharmD clinical pharmacologists, and implementation of user-friendly methods for novel beta-lactams still represent critical issues [97,102-104].

Furthermore, alternative agents should be considered for the treatment of patients with well-documented life-threatening beta-lactam allergies. Fluoroquinolones, aminoglycosides and colistin could be helpful in these cases depending on the susceptibility pattern of Pseudomonas aeruginosa or Acinetobacter baumannii. Besides, in case of life-threatening infections caused by MDR non-fermentative Gram-negatives with limited therapeutic options, desensitization protocols should be taken into consideration.

Finally, it should be mentioned that the current COVID-19 pandemic has led to a worldwide rise in antimicrobial resistance due to the massive disruption of infection control and antimicrobial stewardship measures in COVID ICUs. This caused a remarkable proportion of MDR bacterial co-infections and super-infections (including Pseudomonas aeruginosa and Acinetobacter baumannii) in severe COVID-19 patients [105]. In this scenario of more and more growing antimicrobial resistance, educational programs for improving the culture of knowledge of appropriate antibiotic use and the pivotal role of antimicrobial stewardship should be planned and delivered to young physicians [106].

In recent years, several leading guidelines for the management of Gram-negative infections have been issued by the most important Infectious Disease Societies worldwide [107-109]. Unfortunately, none of these focused on the treatment of IVACs and/or provided extensive recommendations for appropriate place in therapy of novel agents and/or for dosing optimization according to the PK/PD principles. Consequently, the implementation of developed therapeutic algorithms based on susceptibility pattern of non-fermenting Gram-negative isolated pathogens, coupled with the administration of altered dosing strategies of beta-lactams [97], could provide intensive care physicians a useful guidance for maximizing antibiotic treatment in ICU patients affected by IVACs, in order to address three main purposes: (a) to provide a personalized and targeted antibiotic therapy in each critically ill patient affected by HAP/VAP due to Pseudomonas aeruginosa or Acinetobacter baumannii; (b) to appropriately place novel antimicrobial agents in lack of definitive evidence; (c) to consider antimicrobial stewardship strategies for sparing 
the broadest-spectrum antibiotics (namely carbapenems). It is supposed that this strategy could maximize clinical outcome while minimizing resistance development in a challenging scenario as the management of IVACs in ICU patients.

\section{Materials and Methods}

A multidisciplinary task force, composed by one intensive care physician (B.V.), one infectious disease consultant (P.V.), one clinical microbiologist (G.M.R.), and one MD clinical pharmacologist (F.P.) met virtually on several occasions with the intent of developing algorithms for targeted antimicrobial therapy of IVACs caused by Pseudomonas aeruginosa and Acinetobacter baumannii in ICU critically ill patients. IVACs were defined as the presence of a ventilator-associated condition (consisting in a $48 \mathrm{~h}$ stable or decreasing daily minimum positive end-expiratory pressure [PEEP] or $\mathrm{FiO}_{2}$ followed by a rise in PEEP of $3 \mathrm{~cm} \mathrm{H} \mathrm{H}_{2} \mathrm{O}$ or a rise in $\mathrm{FiO}_{2}$ of 0.2 sustained for $48 \mathrm{~h}$ ) coupled with the occurrence of body temperature $<36{ }^{\circ} \mathrm{C}$ or $>38^{\circ} \mathrm{C}$ and the start of at least one antibiotic agent continued for over $96 \mathrm{~h}$. VAP was considered a subgroup of IVACs, consisting in the presence of at least 25 neutrophils / field coupled with positive semi-quantitative/quantitative culture for pathogenic organisms at bronchoalveolar lavage [110]. The definitive agreement for each therapeutic algorithm was reached by the multidisciplinary team after thoroughly discussion based on specific long-standing experience and on the specific expertise of each single member in terms of management of critically ill patients affected by IVACs, in appropriately placing in therapy of the old and novel antimicrobial agents, in implementing appropriate target antibiotic therapy and antimicrobial stewardship strategies in challenging scenarios, in applying traditional and novel microbiological methods and in interpreting microbiological findings and susceptibility test according to the specific clinical scenarios, and in optimizing and individualizing antibiotic dosing regimens according to the specific pathophysiological alterations. Each therapeutic algorithm was designed after that unanimous agreement among the four members of the multidisciplinary team was achieved. Six different scenarios were identified based on the resistance genotype of the pathogens and/or on the antibiotic susceptibility pattern (namely multi-susceptible Pseudomonas aeruginosa, MDR metallo-beta-lactamase-negative Pseudomonas aeruginosa, MDR metallo-beta-lactamase-positive Pseudomonas aeruginosa, carbapenem-susceptible Acinetobacter baumannii, and carbapenem-resistant Acinetobacter baumannii). MDR Pseudomonas aeruginosa isolates were defined according to the classification proposed by Magiorakos et al. [111]. A hierarchical scale was established whenever agreement on multiple therapeutic options was achieved in one specific scenario. Optimized antibiotic dosing schedules were provided as well.

A researcher (M.G.) retrieved the scientific evidence needed for supporting the specific choices included in the algorithms by means of a PubMed-MEDLINE literature search (until October 2021). Key terms were selected antibiotics, HAP, VAP, IVACs, and bacterial pathogens with genotype of resistance and/or antibiotic susceptibility pattern. Quality of evidence was established according to a hierarchical scale of the study design, as reported in the evidence pyramid [112]: randomized controlled trials (RCTs), prospective observational studies, retrospective observational studies, case series, case reports, and in vitro studies. International guidelines issued by the Infectious Disease Society of America and/or by the European Society of Clinical Microbiology and Infectious Diseases, systematic reviews and meta-analyses were also consulted. Consistence between retrieved studies was also considered, by assessing the concordance in clinical outcome of the included studies at each level of the evidence pyramid. Only articles published in English were considered, with a focus mainly on studies published in the last ten years.

\section{Conclusions}

In an era characterized by widespread diffusion of MDR Gram-negative pathogens and continuous increase in antibiotic resistance, the implementation of a multidisciplinary taskforce focusing on targeted therapy in critically ill patients has become a real need. Our 
approach is focused on prompt revision of inappropriate/unnecessary antibiotic therapy, implementation of "carbapenem-sparing" strategies, and PK/PD optimization of antibiotic exposure hopefully guided by real-time TDM whenever feasible. Rational use of broadspectrum antibiotics, especially carbapenems, could represent a powerful strategy for tackling resistance spread in the ICU setting [14]. We believe that this strategy and these algorithms could be helpful in improving clinical outcomes and in avoiding resistance spread in the IVAC setting. The availability of molecular diagnostic tests that can rapidly provide information about the nature of the infecting pathogens and the presence of some relevant resistance determinants will be instrumental to improve antimicrobial stewardship practices based on the proposed algorithms.

Author Contributions: Conceptualization, B.V., G.M.R., F.P. and P.V.; methodology, M.G.; data curation, M.G.; writing-original draft preparation, M.G.; writing-review and editing, B.V., G.M.R., F.P. and P.V. All authors have read and agreed to the published version of the manuscript.

Funding: This research received no external funding.

Institutional Review Board Statement: Not applicable.

Informed Consent Statement: Not applicable.

Data Availability Statement: The data presented in this review are retrieved and summarized from the different publicly available included studies.

Conflicts of Interest: M.G. received personal fees from Angelini; B.V participated in advisory boards and in speaker's bureau for, and received research contracts, contributions and study events from Abbott, Accelerate Diagnostics, Ada, Alifax, Angelini, Becton Dickinson, Bellco, Biomerieux, Biotest, Cepheid, Correvio, Gilead, Menarini, MSD Italia, Nordic Pharma, Pfizer, Shionogi, Thermo Fisher Scientific; G.M.R participated in advisory boards and speaker's bureau for, and received research contracts, contributions and travel grants from Accelerate, Angelini, Arrow, Beckman Biomedical Service, Coulter, Becton-Dickinson, bioMérieux, Cepheid, Hain Life Sciences, Menarini, Meridian, MSD, Nordic Pharma, Pfizer, Qiagen, Q-linea, Qpex, Quidel, Qvella, Roche, Seegene, Set-Lance, Shionogi, Symcel, ThermoFisher, VenatorX, Zambon; F.P participated on the speaker's bureau for Angelini, Basilea Pharmaceutica, Gilead, Hikma, Merck Sharp \& Dohme, Nordic Pharma, Pfizer, and Sanofi Aventis, and in the advisory board for Angelini, Basilea Pharmaceutica, Correvio, Gilead, Merck Sharp \& Dohme, Nordic Pharma, Novartis, Pfizer, and Thermo-Fisher. P.V has served as a consultant for Biomerieux, Gilead, Merck Sharp \& Dohme, Nabriva, Nordic Pharma, Pfizer, ThermoFisher, and Venatorx, and received payment for serving on the speaker's bureau for Correvio, Gilead, Merck Sharp \& Dohme, Nordic Pharma and Pfizer. The authors report no other conflicts of interest in this work.

\section{References}

1. Alberti, C.; Brun-Buisson, C.; Burchardi, H.; Martin, C.; Goodman, S.; Artigas, A.; Sicignano, A.; Palazzo, M.; Moreno, R.; Boulmé, R.; et al. Epidemiology of Sepsis and Infection in ICU Patients from an International Multicentre Cohort Study. Intensive Care Med. 2002, 28, 108-121. [CrossRef] [PubMed]

2. Bassetti, M.; Righi, E.; Vena, A.; Graziano, E.; Russo, A.; Peghin, M. Risk Stratification and Treatment of ICU-Acquired Pneumonia Caused by Multidrug- Resistant/Extensively Drug-Resistant/Pandrug-Resistant Bacteria. Curr. Opin. Crit. Care 2018, 24, 385-393. [CrossRef] [PubMed]

3. Melsen, W.G.; Rovers, M.M.; Groenwold, R.H.H.; Bergmans, D.C.J.J.; Camus, C.; Bauer, T.T.; Hanisch, E.W.; Klarin, B.; Koeman, M.; Krueger, W.A.; et al. Attributable Mortality of Ventilator-Associated Pneumonia: A Meta-Analysis of Individual Patient Data from Randomised Prevention Studies. Lancet Infect. Dis. 2013, 13, 665-671. [CrossRef]

4. Vincent, J.-L.; Sakr, Y.; Singer, M.; Martin-Loeches, I.; Machado, F.R.; Marshall, J.C.; Finfer, S.; Pelosi, P.; Brazzi, L.; Aditianingsih, D.; et al. Prevalence and Outcomes of Infection Among Patients in Intensive Care Units in 2017. JAMA 2020, 323, 1478-1487. [CrossRef]

5. Bekaert, M.; Timsit, J.-F.; Vansteelandt, S.; Depuydt, P.; Vésin, A.; Garrouste-Orgeas, M.; Decruyenaere, J.; Clec'h, C.; Azoulay, E.; Benoit, D.; et al. Attributable Mortality of Ventilator-Associated Pneumonia: A Reappraisal Using Causal Analysis. Am. J. Respir. Crit. Care Med. 2011, 184, 1133-1139. [CrossRef] [PubMed]

6. Kidd, J.M.; Kuti, J.L.; Nicolau, D.P. Novel Pharmacotherapy for the Treatment of Hospital-Acquired and Ventilator-Associated Pneumonia Caused by Resistant Gram-Negative Bacteria. Expert Opin. Pharmacother. 2018, 19, 397-408. [CrossRef] 
7. Garnacho-Montero, J.; Timsit, J.-F. Managing Acinetobacter Baumannii Infections. Curr. Opin. Infect. Dis. 2019, 32, 69-76. [CrossRef] [PubMed]

8. Amaya-Villar, R.; Garnacho-Montero, J. How Should We Treat Acinetobacter Pneumonia? Curr. Opin. Crit. Care 2019, 25, 465-472. [CrossRef]

9. Giannella, M.; Bartoletti, M.; Gatti, M.; Viale, P. Advances in the Therapy of Bacterial Bloodstream Infections. Clin. Microbiol Infect. 2020, 26, 158-167. [CrossRef]

10. ECDC. Antimicrobial Resistance in the EU/EEA (EARS-Net)—Annual Epidemiological Report for 2019; European Centre for Disease Prevention and Control: Solna, Sweden, 2020.

11. Evans, L.; Rhodes, A.; Alhazzani, W.; Antonelli, M.; Coopersmith, C.M.; French, C.; Machado, F.R.; Mcintyre, L.; Ostermann, M.; Prescott, H.C.; et al. Surviving Sepsis Campaign: International Guidelines for Management of Sepsis and Septic Shock 2021. Intensive Care Med. 2021, 47, 1181-1247. [CrossRef]

12. Poole, S.; Clark, T.W. Rapid Syndromic Molecular Testing in Pneumonia: The Current Landscape and Future Potential. J. Infect. 2020, 80, 1-7. [CrossRef]

13. Crémet, L.; Gaborit, B.; Bouras, M.; Drumel, T.; Guillotin, F.; Poulain, C.; Persyn, E.; Lakhal, K.; Rozec, B.; Vibet, M.-A.; et al. Evaluation of the FilmArray ${ }^{\circledR}$ Pneumonia Plus Panel for Rapid Diagnosis of Hospital-Acquired Pneumonia in Intensive Care Unit Patients. Front. Microbiol. 2020, 11, 2080. [CrossRef] [PubMed]

14. Timsit, J.-F.; Bassetti, M.; Cremer, O.; Daikos, G.; de Waele, J.; Kallil, A.; Kipnis, E.; Kollef, M.; Laupland, K.; Paiva, J.-A.; et al. Rationalizing Antimicrobial Therapy in the ICU: A Narrative Review. Intensive Care Med. 2019, 45, 172-189. [CrossRef]

15. Kalil, A.C.; Metersky, M.L.; Klompas, M.; Muscedere, J.; Sweeney, D.A.; Palmer, L.B.; Napolitano, L.M.; O'Grady, N.P.; Bartlett, J.G.; Carratalà, J.; et al. Management of Adults With Hospital-Acquired and Ventilator-Associated Pneumonia: 2016 Clinical Practice Guidelines by the Infectious Diseases Society of America and the American Thoracic Society. Clin. Infect. Dis. 2016, 63, e61-e111. [CrossRef]

16. Jaccard, C.; Troillet, N.; Harbarth, S.; Zanetti, G.; Aymon, D.; Schneider, R.; Chiolero, R.; Ricou, B.; Romand, J.; Huber, O.; et al. Prospective Randomized Comparison of Imipenem-Cilastatin and Piperacillin-Tazobactam in Nosocomial Pneumonia or Peritonitis. Antimicrob. Agents Chemother. 1998, 42, 2966-2972. [CrossRef] [PubMed]

17. Joshi, M.; Bernstein, J.; Solomkin, J.; Wester, B.A.; Kuye, O. Piperacillin/Tazobactam plus Tobramycin versus Ceftazidime plus Tobramycin for the Treatment of Patients with Nosocomial Lower Respiratory Tract Infection. Piperacillin/Tazobactam Nosocomial Pneumonia Study Group. J. Antimicrob. Chemother. 1999, 43, 389-397. [CrossRef] [PubMed]

18. Babich, T.; Naucler, P.; Valik, J.K.; Giske, C.G.; Benito, N.; Cardona, R.; Rivera, A.; Pulcini, C.; Abdel Fattah, M.; Haquin, J.; et al. Ceftazidime, Carbapenems, or Piperacillin-Tazobactam as Single Definitive Therapy for Pseudomonas Aeruginosa Bloodstream Infection: A Multisite Retrospective Study. Clin. Infect. Dis. 2020, 70, 2270-2280. [CrossRef]

19. Su, T.-Y.; Ye, J.-J.; Yang, C.-C.; Huang, C.-T.; Chia, J.-H.; Lee, M.-H. Influence of Borderline Cefepime MIC on the Outcome of Cefepime-Susceptible Pseudomonas aeruginosa Bacteremia Treated with a Maximal Cefepime Dose: A Hospital-Based Retrospective Study. Ann. Clin. Microbiol. Antimicrob. 2017, 16, 52. [CrossRef]

20. Ratliff, A.R.; Gentry, C.A.; Williams, R.J. A Propensity Score-Matched Analysis of the Impact of Minimum Inhibitory Concentration on Mortality in Patients with Pseudomonas Aeruginosa Bacteremia Treated with Cefepime or Ceftazidime. Diagn. Microbiol. Infect. Dis 2017, 87, 376-381. [CrossRef]

21. Vardakas, K.Z.; Voulgaris, G.L.; Maliaros, A.; Samonis, G.; Falagas, M.E. Prolonged versus Short-Term Intravenous Infusion of Antipseudomonal $\beta$-Lactams for Patients with Sepsis: A Systematic Review and Meta-Analysis of Randomised Trials. Lancet Infect. Dis 2018, 18, 108-120. [CrossRef]

22. Gatti, M.; Cojutti, P.G.; Pascale, R.; Tonetti, T.; Laici, C.; Dell'Olio, A.; Siniscalchi, A.; Giannella, M.; Viale, P.; Pea, F. Assessment of a PK/PD Target of Continuous Infusion Beta-Lactams Useful for Preventing Microbiological Failure and/or Resistance Development in Critically Ill Patients Affected by Documented Gram-Negative Infections. Antibiotics 2021, 10, 1311. [CrossRef]

23. Horcajada, J.P.; Montero, M.; Oliver, A.; Sorlí, L.; Luque, S.; Gómez-Zorrilla, S.; Benito, N.; Grau, S. Epidemiology and Treatment of Multidrug-Resistant and Extensively Drug-Resistant Pseudomonas Aeruginosa Infections. Clin. Microbiol. Rev. 2019, 32, e00031-19. [CrossRef] [PubMed]

24. Kollef, M.H.; Nováček, M.; Kivistik, Ü.; Réa-Neto, Á.; Shime, N.; Martin-Loeches, I.; Timsit, J.-F.; Wunderink, R.G.; Bruno, C.J.; Huntington, J.A.; et al. Ceftolozane-Tazobactam versus Meropenem for Treatment of Nosocomial Pneumonia (ASPECT-NP): A Randomised, Controlled, Double-Blind, Phase 3, Non-Inferiority Trial. Lancet Infect. Dis. 2019, 19, 1299-1311. [CrossRef]

25. Pogue, J.M.; Kaye, K.S.; Veve, M.P.; Patel, T.S.; Gerlach, A.T.; Davis, S.L.; Puzniak, L.A.; File, T.M.; Olson, S.; Dhar, S.; et al. Ceftolozane/Tazobactam vs Polymyxin or Aminoglycoside-Based Regimens for the Treatment of Drug-Resistant Pseudomonas Aeruginosa. Clin. Infect. Dis 2020, 71, 304-310. [CrossRef] [PubMed]

26. Bassetti, M.; Castaldo, N.; Cattelan, A.; Mussini, C.; Righi, E.; Tascini, C.; Menichetti, F.; Mastroianni, C.M.; Tumbarello, M.; Grossi, P.; et al. Ceftolozane/Tazobactam for the Treatment of Serious Pseudomonas aeruginosa Infections: A Multicentre Nationwide Clinical Experience. Int. J. Antimicrob. Agents 2019, 53, 408-415. [CrossRef] [PubMed]

27. Balandin, B.; Ballesteros, D.; Ruiz de Luna, R.; López-Vergara, L.; Pintado, V.; Sancho-González, M.; Soriano-Cuesta, C.; PérezPedrero, M.J.; Asensio-Martín, M.J.; Fernández-Simón, I.; et al. Multicenter Study of Ceftolozane/Tazobactam for Treatment of Pseudomonas Aeruginosa Infections in Critically Ill Patients. Int. J. Antimicrob. Agents 2021, 57, 106270. [CrossRef] 
28. Fernández-Cruz, A.; Alba, N.; Semiglia-Chong, M.A.; Padilla, B.; Rodríguez-Macías, G.; Kwon, M.; Cercenado, E.; Chamorrode-Vega, E.; Machado, M.; Pérez-Lago, L.; et al. A Case-Control Study of Real-Life Experience with Ceftolozane-Tazobactam in Patients with Hematologic Malignancy and Pseudomonas Aeruginosa Infection. Antimicrob. Agents Chemother. 2019, 63, e02340-18. [CrossRef] [PubMed]

29. Gallagher, J.C.; Satlin, M.J.; Elabor, A.; Saraiya, N.; McCreary, E.K.; Molnar, E.; El-Beyrouty, C.; Jones, B.M.; Dixit, D.; Heil, E.L.; et al. Ceftolozane-Tazobactam for the Treatment of Multidrug-Resistant Pseudomonas aeruginosa Infections: A Multicenter Study. Open Forum Infect. Dis. 2018, 5, ofy280. [CrossRef]

30. Rodríguez-Núñez, O.; Periañez-Parraga, L.; Oliver, A.; Munita, J.M.; Boté, A.; Gasch, O.; Nuvials, X.; Dinh, A.; Shaw, R.; Lomas, J.M.; et al. Higher MICs (>2 Mg/L) Predict 30-Day Mortality in Patients With Lower Respiratory Tract Infections Caused by Multidrug- and Extensively Drug-Resistant Pseudomonas aeruginosa Treated With Ceftolozane/Tazobactam. Open Forum Infect. Dis. 2019, 6, ofz416. [CrossRef]

31. Munita, J.M.; Aitken, S.L.; Miller, W.R.; Perez, F.; Rosa, R.; Shimose, L.A.; Lichtenberger, P.N.; Abbo, L.M.; Jain, R.; Nigo, M.; et al. Multicenter Evaluation of Ceftolozane/Tazobactam for Serious Infections Caused by Carbapenem-Resistant Pseudomonas aeruginosa. Clin. Infect. Dis. 2017, 65, 158-161. [CrossRef]

32. Haidar, G.; Philips, N.J.; Shields, R.K.; Snyder, D.; Cheng, S.; Potoski, B.A.; Doi, Y.; Hao, B.; Press, E.G.; Cooper, V.S.; et al. Ceftolozane-Tazobactam for the Treatment of Multidrug-Resistant Pseudomonas aeruginosa Infections: Clinical Effectiveness and Evolution of Resistance. Clin. Infect. Dis. 2017, 65, 110-120. [CrossRef] [PubMed]

33. Bosaeed, M.; Ahmad, A.; Alali, A.; Mahmoud, E.; Alswidan, L.; Alsaedy, A.; Aljuhani, S.; Alalwan, B.; Alshamrani, M.; Alothman, A. Experience With Ceftolozane-Tazobactam for the Treatment of Serious Pseudomonas aeruginosa Infections in Saudi Tertiary Care Center. Infect. Dis. 2020, 13, 1178633720905977. [CrossRef] [PubMed]

34. Díaz-Cañestro, M.; Periañez, L.; Mulet, X.; Martin-Pena, M.L.; Fraile-Ribot, P.A.; Ayestarán, I.; Colomar, A.; Nuñez, B.; Maciá, M.; Novo, A.; et al. Ceftolozane/Tazobactam for the Treatment of Multidrug Resistant Pseudomonas aeruginosa: Experience from the Balearic Islands. Eur. J. Clin. Microbiol. Infect. Dis. 2018, 37, 2191-2200. [CrossRef] [PubMed]

35. Escolà-Vergé, L.; Pigrau, C.; Los-Arcos, I.; Arévalo, Á.; Viñado, B.; Campany, D.; Larrosa, N.; Nuvials, X.; Ferrer, R.; Len, O.; et al. Ceftolozane/Tazobactam for the Treatment of XDR Pseudomonas aeruginosa Infections. Infection 2018, 46, 461-468. [CrossRef] [PubMed]

36. Xipell, M.; Paredes, S.; Fresco, L.; Bodro, M.; Marco, F.; Martínez, J.A.; Soriano, A. Clinical Experience with Ceftolozane/Tazobactam in Patients with Serious Infections Due to Resistant Pseudomonas aeruginosa. J. Glob. Antimicrob. Resist. 2018, 13, 165-170. [CrossRef]

37. Castón, J.J.; De la Torre, Á.; Ruiz-Camps, I.; Sorlí, M.L.; Torres, V.; Torre-Cisneros, J. Salvage Therapy with Ceftolozane-Tazobactam for Multidrug-Resistant Pseudomonas aeruginosa Infections. Antimicrob. Agents Chemother. 2017, 61, e02136-16. [CrossRef]

38. Dinh, A.; Wyplosz, B.; Kernéis, S.; Lebeaux, D.; Bouchand, F.; Duran, C.; Béraud, G.; Lazaro, P.; Davido, B.; Hénard, S.; et al. Use of Ceftolozane/Tazobactam as Salvage Therapy for Infections Due to Extensively Drug-Resistant Pseudomonas aeruginosa. Int. J. Antimicrob. Agents 2017, 49, 782-783. [CrossRef]

39. Gelfand, M.S.; Cleveland, K.O. Ceftolozane/Tazobactam Therapy of Respiratory Infections Due to Multidrug-Resistant Pseudomonas aeruginosa. Clin. Infect. Dis. 2015, 61, 853-855. [CrossRef]

40. Hakki, M.; Lewis, J.S. Ceftolozane-Tazobactam Therapy for Multidrug-Resistant Pseudomonas aeruginosa Infections in Patients with Hematologic Malignancies and Hematopoietic-Cell Transplant Recipients. Infection 2018, 46, 431-434. [CrossRef]

41. Bassetti, M.; Echols, R.; Matsunaga, Y.; Ariyasu, M.; Doi, Y.; Ferrer, R.; Lodise, T.P.; Naas, T.; Niki, Y.; Paterson, D.L.; et al. Efficacy and Safety of Cefiderocol or Best Available Therapy for the Treatment of Serious Infections Caused by Carbapenem-Resistant Gram-Negative Bacteria (CREDIBLE-CR): A Randomised, Open-Label, Multicentre, Pathogen-Focused, Descriptive, Phase 3 Trial. Lancet Infect. Dis. 2021, 21, 226-240. [CrossRef]

42. Wunderink, R.G.; Matsunaga, Y.; Ariyasu, M.; Clevenbergh, P.; Echols, R.; Kaye, K.S.; Kollef, M.; Menon, A.; Pogue, J.M.; Shorr, A.F.; et al. Cefiderocol versus High-Dose, Extended-Infusion Meropenem for the Treatment of Gram-Negative Nosocomial Pneumonia (APEKS-NP): A Randomised, Double-Blind, Phase 3, Non-Inferiority Trial. Lancet Infect. Dis. 2021, $21,213-225$. [CrossRef]

43. Delgado-Valverde, M.; Conejo, M.D.C.; Serrano, L.; Fernández-Cuenca, F.; Pascual, Á. Activity of Cefiderocol against HighRisk Clones of Multidrug-Resistant Enterobacterales, Acinetobacter Baumannii, Pseudomonas aeruginosa and Stenotrophomonas Maltophilia. J. Antimicrob. Chemother. 2020, 75, 1840-1849. [CrossRef]

44. Mushtaq, S.; Sadouki, Z.; Vickers, A.; Livermore, D.M.; Woodford, N. In Vitro Activity of Cefiderocol, a Siderophore Cephalosporin, against Multidrug-Resistant Gram-Negative Bacteria. Antimicrob. Agents Chemother. 2020, 64. [CrossRef]

45. Kazmierczak, K.M.; Tsuji, M.; Wise, M.G.; Hackel, M.; Yamano, Y.; Echols, R.; Sahm, D.F. In Vitro Activity of Cefiderocol, a Siderophore Cephalosporin, against a Recent Collection of Clinically Relevant Carbapenem-Non-Susceptible Gram-Negative Bacilli, Including Serine Carbapenemase- and Metallo- $\beta$-Lactamase-Producing Isolates (SIDERO-WT-2014 Study). Int. J Antimicrob. Agents 2019, 53, 177-184. [CrossRef]

46. Pilmis, B.; Petitjean, G.; Lesprit, P.; Lafaurie, M.; El Helali, N.; Le Monnier, A.; on behalf the ATB PK/PD Study Group. Continuous Infusion of Ceftolozane/Tazobactam Is Associated with a Higher Probability of Target Attainment in Patients Infected with Pseudomonas Aeruginosa. Eur. J. Clin. Microbiol. Infect. Dis. 2019, 38, 1457-1461. [CrossRef] [PubMed] 
47. Tamma, P.D.; Beisken, S.; Bergman, Y.; Posch, A.E.; Avdic, E.; Sharara, S.L.; Cosgrove, S.E.; Simner, P.J. Modifiable Risk Factors for the Emergence of Ceftolozane-Tazobactam Resistance. Clin. Infect. Dis. 2020, 73, e4599-e4606. [CrossRef] [PubMed]

48. Torres, A.; Zhong, N.; Pachl, J.; Timsit, J.-F.; Kollef, M.; Chen, Z.; Song, J.; Taylor, D.; Laud, P.J.; Stone, G.G.; et al. CeftazidimeAvibactam versus Meropenem in Nosocomial Pneumonia, Including Ventilator-Associated Pneumonia (REPROVE): A Randomised, Double-Blind, Phase 3 Non-Inferiority Trial. Lancet Infect. Dis. 2018, 18, 285-295. [CrossRef]

49. Jorgensen, S.C.J.; Trinh, T.D.; Zasowski, E.J.; Lagnf, A.M.; Bhatia, S.; Melvin, S.M.; Steed, M.E.; Simon, S.P.; Estrada, S.J.; Morrisette, T.; et al. Real-World Experience with Ceftazidime-Avibactam for Multidrug-Resistant Gram-Negative Bacterial Infections. Open Forum Infect. Dis. 2019, 6, ofz522. [CrossRef] [PubMed]

50. Vena, A.; Giacobbe, D.R.; Castaldo, N.; Cattelan, A.; Mussini, C.; Luzzati, R.; Rosa, F.G.D.; Del Puente, F.; Mastroianni, C.M.; Cascio, A.; et al. Clinical Experience with Ceftazidime-Avibactam for the Treatment of Infections Due to Multidrug-Resistant Gram-Negative Bacteria Other than Carbapenem-Resistant Enterobacterales. Antibiotics 2020, 9, E71. [CrossRef]

51. Rodríguez-Núñez, O.; Ripa, M.; Morata, L.; de la Calle, C.; Cardozo, C.; Fehér, C.; Pellicé, M.; Valcárcel, A.; Puerta-Alcalde, P.; Marco, F.; et al. Evaluation of Ceftazidime/Avibactam for Serious Infections Due to Multidrug-Resistant and Extensively Drug-Resistant Pseudomonas Aeruginosa. J. Glob. Antimicrob. Resist. 2018, 15, 136-139. [CrossRef]

52. Santevecchi, B.A.; Smith, T.T.; MacVane, S.H. Clinical Experience with Ceftazidime/Avibactam for Treatment of AntibioticResistant Organisms Other than Klebsiella Pneumoniae. Int. J. Antimicrob. Agents 2018, 51, 629-635. [CrossRef]

53. Xipell, M.; Bodro, M.; Marco, F.; Losno, R.A.; Cardozo, C.; Soriano, A. Clinical Experience with Ceftazidime/Avibactam in Patients with Severe Infections, Including Meningitis and Lung Abscesses, Caused by Extensively Drug-Resistant Pseudomonas Aeruginosa. Int. J. Antimicrob. Agents 2017, 49, 266-268. [CrossRef]

54. Recio, R.; Villa, J.; Viedma, E.; Orellana, M.Á.; Lora-Tamayo, J.; Chaves, F. Bacteraemia Due to Extensively Drug-Resistant Pseudomonas Aeruginosa Sequence Type 235 High-Risk Clone: Facing the Perfect Storm. Int J. Antimicrob. Agents 2018, 52, 172-179. [CrossRef] [PubMed]

55. Papp-Wallace, K.M.; Zeiser, E.T.; Becka, S.A.; Park, S.; Wilson, B.M.; Winkler, M.L.; D’Souza, R.; Singh, I.; Sutton, G.; Fouts, D.E.; et al. Ceftazidime-Avibactam in Combination With Fosfomycin: A Novel Therapeutic Strategy Against Multidrug-Resistant Pseudomonas aeruginosa. J. Infect. Dis. 2019, 220, 666-676. [CrossRef] [PubMed]

56. Avery, L.M.; Sutherland, C.A.; Nicolau, D.P. In Vitro Investigation of Synergy among Fosfomycin and Parenteral Antimicrobials against Carbapenemase-Producing Enterobacteriaceae. Diagn. Microbiol. Infect. Dis. 2019, 95, 216-220. [CrossRef] [PubMed]

57. Mikhail, S.; Singh, N.B.; Kebriaei, R.; Rice, S.A.; Stamper, K.C.; Castanheira, M.; Rybak, M.J. Evaluation of the Synergy of Ceftazidime-Avibactam in Combination with Meropenem, Amikacin, Aztreonam, Colistin, or Fosfomycin against WellCharacterized Multidrug-Resistant Klebsiella Pneumoniae and Pseudomonas aeruginosa. Antimicrob. Agents Chemother. $2019,63$. [CrossRef] [PubMed]

58. Tumbarello, M.; Raffaelli, F.; Giannella, M.; Mantengoli, E.; Mularoni, A.; Venditti, M.; De Rosa, F.G.; Sarmati, L.; Bassetti, M.; Brindicci, G.; et al. Ceftazidime-Avibactam Use for KPC-Kp Infections: A Retrospective Observational Multicenter Study. Clin. Infect. Dis. 2021, 73, 1664-1676. [CrossRef]

59. Gatti, M.; Bartoletti, M.; Cojutti, P.G.; Gaibani, P.; Conti, M.; Giannella, M.; Viale, P.; Pea, F. A Descriptive Case Series of PK/PD Target Attainment and Microbiological Outcome in Critically Ill Patients with Documented Severe XDR Acinetobacter Baumannii BSI and/or VAP Treated with Cefiderocol. J. Glob. Antimicrob. Resist. 2021, 27, 294-298. [CrossRef]

60. Nicolau, D.P.; Siew, L.; Armstrong, J.; Li, J.; Edeki, T.; Learoyd, M.; Das, S. Phase 1 Study Assessing the Steady-State Concentration of Ceftazidime and Avibactam in Plasma and Epithelial Lining Fluid Following Two Dosing Regimens. J. Antimicrob. Chemother. 2015, 70, 2862-2869. [CrossRef]

61. Katsube, T.; Nicolau, D.P.; Rodvold, K.A.; Wunderink, R.G.; Echols, R.; Matsunaga, Y.; Menon, A.; Portsmouth, S.; Wajima, T. Intrapulmonary Pharmacokinetic Profile of Cefiderocol in Mechanically Ventilated Patients with Pneumonia. J. Antimicrob. Chemother. 2021, 76, 2902-2905. [CrossRef]

62. Katsube, T.; Saisho, Y.; Shimada, J.; Furuie, H. Intrapulmonary Pharmacokinetics of Cefiderocol, a Novel Siderophore Cephalosporin, in Healthy Adult Subjects. J. Antimicrob. Chemother. 2019, 74, 1971-1974. [CrossRef] [PubMed]

63. Jacobs, M.R.; Abdelhamed, A.M.; Good, C.E.; Rhoads, D.D.; Hujer, K.M.; Hujer, A.M.; Domitrovic, T.N.; Rudin, S.D.; Richter, S.S.; van Duin, D.; et al. ARGONAUT-I: Activity of Cefiderocol (S-649266), a Siderophore Cephalosporin, against Gram-Negative Bacteria, Including Carbapenem-Resistant Nonfermenters and Enterobacteriaceae with Defined Extended-Spectrum $\beta$-Lactamases and Carbapenemases. Antimicrob. Agents Chemother. 2019, 63. [CrossRef] [PubMed]

64. Albiero, J.; Mazucheli, J.; Barros, J.P.D.R.; Szczerepa, M.M.D.A.; Nishiyama, S.A.B.; Carrara-Marroni, F.E.; Sy, S.; Fidler, M.; Sy, S.K.B.; Tognim, M.C.B. Pharmacodynamic Attainment of the Synergism of Meropenem and Fosfomycin Combination against Pseudomonas aeruginosa Producing Metallo- $\beta$-Lactamase. Antimicrob. Agents Chemother. 2019, 63, e00126-19. [CrossRef]

65. Vardakas, K.Z.; Voulgaris, G.L.; Samonis, G.; Falagas, M.E. Inhaled Colistin Monotherapy for Respiratory Tract Infections in Adults without Cystic Fibrosis: A Systematic Review and Meta-Analysis. Int. J. Antimicrob. Agents 2018, 51, 1-9. [CrossRef] [PubMed]

66. Alamarat, Z.I.; Babic, J.; Tran, T.T.; Wootton, S.H.; Dinh, A.Q.; Miller, W.R.; Hanson, B.; Wanger, A.; Gary, J.L.; Arias, C.A.; et al. Long-Term Compassionate Use of Cefiderocol To Treat Chronic Osteomyelitis Caused by Extensively Drug-Resistant Pseudomonas aeruginosa and Extended-Spectrum- $\beta$-Lactamase-Producing Klebsiella Pneumoniae in a Pediatric Patient. Antimicrob. Agents Chemother. 2020, 64, e01872-19. [CrossRef] 
67. Zingg, S.; Nicoletti, G.J.; Kuster, S.; Junker, M.; Widmer, A.; Egli, A.; Hinic, V.; Sendi, P.; Battegay, M.; Bättig, V.; et al. Cefiderocol for Extensively Drug-Resistant Gram-Negative Bacterial Infections: Real-World Experience From a Case Series and Review of the Literature. Open Forum Infect. Dis. 2020, 7, ofaa185. [CrossRef]

68. Cojutti, P.G.; Gatti, M.; Rinaldi, M.; Tonetti, T.; Laici, C.; Mega, C.; Siniscalchi, A.; Giannella, M.; Viale, P.; Pea, F. Impact of Maximizing Css/MIC Ratio on Efficacy of Continuous Infusion Meropenem Against Documented Gram-Negative Infections in Critically Ill Patients and Population Pharmacokinetic/Pharmacodynamic Analysis to Support Treatment Optimization. Front. Pharmacol. 2021, 12, 781892. [CrossRef]

69. Garnacho-Montero, J.; Ortiz-Leyba, C.; Jiménez-Jiménez, F.J.; Barrero-Almodóvar, A.E.; García-Garmendia, J.L.; Bernabeu-WittelI, M.; Gallego-Lara, S.L.; Madrazo-Osuna, J. Treatment of Multidrug-Resistant Acinetobacter Baumannii Ventilator-Associated Pneumonia (VAP) with Intravenous Colistin: A Comparison with Imipenem-Susceptible VAP. Clin. Infect. Dis. 2003, 36, 1111-1118. [CrossRef]

70. Kallel, H.; Hergafi, L.; Bahloul, M.; Hakim, A.; Dammak, H.; Chelly, H.; Hamida, C.B.; Chaari, A.; Rekik, N.; Bouaziz, M. Safety and Efficacy of Colistin Compared with Imipenem in the Treatment of Ventilator-Associated Pneumonia: A Matched Case-Control Study. Intensive Care Med. 2007, 33, 1162-1167. [CrossRef]

71. Wang, D. Experience with Extended-Infusion Meropenem in the Management of Ventilator-Associated Pneumonia Due to Multidrug-Resistant Acinetobacter Baumannii. Int. J. Antimicrob. Agents 2009, 33, 290-291. [CrossRef] [PubMed]

72. Ikonomidis, A.; Pournaras, S.; Maniatis, A.N.; Legakis, N.J.; Tsakris, A. Discordance of Meropenem versus Imipenem Activity against Acinetobacter Baumannii. Int. J. Antimicrob. Agents 2006, 28, 376-377. [CrossRef] [PubMed]

73. Mezzatesta, M.L.; Trovato, G.; Gona, F.; Nicolosi, V.M.; Nicolosi, D.; Carattoli, A.; Fadda, G.; Nicoletti, G.; Stefani, S. In Vitro Activity of Tigecycline and Comparators against Carbapenem-Susceptible and Resistant Acinetobacter Baumannii Clinical Isolates in Italy. Ann. Clin. Microbiol. Antimicrob. 2008, 7, 4. [CrossRef] [PubMed]

74. Guzek, A.; Korzeniewski, K.; Nitsch-Osuch, A.; Rybicki, Z.; Prokop, E. In Vitro Sensitivity of Acinetobacter Baumannii and Pseudomonas aeruginosa to Carbapenems among Intensive Care Unit Patients. Adv. Exp. Med. Biol. 2013, 788, 109-116. [CrossRef] [PubMed]

75. Jones, R.N.; Sader, H.S.; Fritsche, T.R. Comparative Activity of Doripenem and Three Other Carbapenems Tested against GramNegative Bacilli with Various Beta-Lactamase Resistance Mechanisms. Diagn. Microbiol. Infect. Dis. 2005, 52, 71-74. [CrossRef] [PubMed]

76. Bavaro, D.F.; Belati, A.; Diella, L.; Stufano, M.; Romanelli, F.; Scalone, L.; Stolfa, S.; Ronga, L.; Maurmo, L.; Dell'Aera, M.; et al. Cefiderocol-Based Combination Therapy for "Difficult-to-Treat" Gram-Negative Severe Infections: Real-Life Case Series and Future Perspectives. Antibiotics 2021, 10, 652. [CrossRef]

77. Falcone, M.; Tiseo, G.; Nicastro, M.; Leonildi, A.; Vecchione, A.; Casella, C.; Forfori, F.; Malacarne, P.; Guarracino, F.; Barnini, S.; et al. Cefiderocol as Rescue Therapy for Acinetobacter Baumannii and Other Carbapenem-Resistant Gram-Negative Infections in ICU Patients. Clin. Infect. Dis 2020, 72, 2021-2024. [CrossRef]

78. Trecarichi, E.M.; Quirino, A.; Scaglione, V.; Longhini, F.; Garofalo, E.; Bruni, A.; Biamonte, E.; Lionello, R.; Serapide, F.; Mazzitelli, M.; et al. Successful Treatment with Cefiderocol for Compassionate Use in a Critically Ill Patient with XDR Acinetobacter Baumannii and KPC-Producing Klebsiella Pneumoniae: A Case Report. J. Antimicrob. Chemother. 2019, 74, $3399-3401$. [CrossRef]

79. Hackel, M.A.; Tsuji, M.; Yamano, Y.; Echols, R.; Karlowsky, J.A.; Sahm, D.F. In Vitro Activity of the Siderophore Cephalosporin, Cefiderocol, against a Recent Collection of Clinically Relevant Gram-Negative Bacilli from North America and Europe, Including Carbapenem-Nonsusceptible Isolates (SIDERO-WT-2014 Study). Antimicrob. Agents Chemother. 2017, 61, e00093-17. [CrossRef]

80. Betrosian, A.P.; Frantzeskaki, F.; Xanthaki, A.; Georgiadis, G. High-Dose Ampicillin-Sulbactam as an Alternative Treatment of Late-Onset VAP from Multidrug-Resistant Acinetobacter baumannii. Scand. J. Infect. Dis. 2007, 39, 38-43. [CrossRef]

81. Betrosian, A.P.; Frantzeskaki, F.; Xanthaki, A.; Douzinas, E.E. Efficacy and Safety of High-Dose Ampicillin/Sulbactam vs. Colistin as Monotherapy for the Treatment of Multidrug Resistant Acinetobacter Baumannii Ventilator-Associated Pneumonia. J. Infect. 2008, 56, 432-436. [CrossRef]

82. Mellon, G.; Clec'h, C.; Picard, B.; Cohen, Y.; Jauréguy, F. Postsurgical Meningitis Due to Multiresistant Acinetobacter baumannii Successfully Treated with High Doses of Ampicillin/Sulbactam Combined with Rifampicin and Fosfomycin. J. Infect. Chemother. 2012, 18, 958-960. [CrossRef] [PubMed]

83. Mohd Sazlly Lim, S.; Heffernan, A.J.; Roberts, J.A.; Sime, F.B. Semi-Mechanistic PK/PD Modelling of Fosfomycin and Sulbactam Combination against Carbapenem-Resistant Acinetobacter baumannii. Antimicrob. Agents Chemother. 2021, 65, e02472-20. [CrossRef]

84. Kuo, S.-C.; Lee, Y.-T.; Yang, S.-P.; Chen, C.-P.; Chen, T.-L.; Hsieh, S.-L.; Siu, L.-K.; Fung, C.-P. Eradication of Multidrug-Resistant Acinetobacter baumannii from the Respiratory Tract with Inhaled Colistin Methanesulfonate: A Matched Case-Control Study. Clin. Microbiol. Infect. 2012, 18, 870-876. [CrossRef] [PubMed]

85. Bassetti, M.; Vena, A.; Croxatto, A.; Righi, E.; Guery, B. How to Manage Pseudomonas Aeruginosa Infections. Drugs Context 2018, 7, 212527. [CrossRef] [PubMed]

86. Caro, L.; Nicolau, D.P.; De Waele, J.J.; Kuti, J.L.; Larson, K.B.; Gadzicki, E.; Yu, B.; Zeng, Z.; Adedoyin, A.; Rhee, E.G. Lung Penetration, Bronchopulmonary Pharmacokinetic/Pharmacodynamic Profile and Safety of $3 \mathrm{~g}$ of Ceftolozane/Tazobactam Administered to Ventilated, Critically Ill Patients with Pneumonia. J. Antimicrob. Chemother. 2020, 75, 1546-1553. [CrossRef] [PubMed] 
87. Gatti, M.; Pea, F. Continuous versus Intermittent Infusion of Antibiotics in Gram-Negative Multidrug-Resistant Infections. Curr. Opin. Infect. Dis. 2021, 34, 737-747. [CrossRef]

88. Lodise, T.P.; Lomaestro, B.; Drusano, G.L. Piperacillin-Tazobactam for Pseudomonas Aeruginosa Infection: Clinical Implications of an Extended-Infusion Dosing Strategy. Clin. Infect. Dis 2007, 44, 357-363. [CrossRef] [PubMed]

89. Boisson, M.; Jacobs, M.; Grégoire, N.; Gobin, P.; Marchand, S.; Couet, W.; Mimoz, O. Comparison of Intrapulmonary and Systemic Pharmacokinetics of Colistin Methanesulfonate (CMS) and Colistin after Aerosol Delivery and Intravenous Administration of CMS in Critically Ill Patients. Antimicrob. Agents Chemother. 2014, 58, 7331-7339. [CrossRef]

90. Benítez-Cano, A.; de Antonio-Cuscó, M.; Luque, S.; Sorlí, L.; Carazo, J.; Ramos, I.; Bermejo, S.; Campillo, N.; Horcajada, J.P.; Samsó, E.; et al. Systemic Pharmacokinetics and Safety of High Doses of Nebulized Colistimethate Sodium in Critically Ill Patients with Hospital-Acquired and Ventilator-Associated Pneumonia. J. Antimicrob. Chemother. 2019, 74, 3268-3273. [CrossRef]

91. Biagi, M.; Butler, D.; Tan, X.; Qasmieh, S.; Wenzler, E. A Breath of Fresh Air in the Fog of Antimicrobial Resistance: Inhaled Polymyxins for Gram-Negative Pneumonia. Antibiotics 2019, 8, E27. [CrossRef]

92. Isler, B.; Doi, Y.; Bonomo, R.A.; Paterson, D.L. New Treatment Options against Carbapenem-Resistant Acinetobacter baumannii Infections. Antimicrob. Agents Chemother. 2019, 63, e01110-18. [CrossRef] [PubMed]

93. Heil, E.L.; Tamma, P.D. Cefiderocol: The Trojan Horse Has Arrived but Will Troy Fall? Lancet Infect. Dis. 2021, 21, 153-155. [CrossRef]

94. Matzi, V.; Lindenmann, J.; Porubsky, C.; Kugler, S.A.; Maier, A.; Dittrich, P.; Smolle-Jüttner, F.M.; Joukhadar, C. Extracellular Concentrations of Fosfomycin in Lung Tissue of Septic Patients. J. Antimicrob. Chemother. 2010, 65, 995-998. [CrossRef]

95. Russo, A.; Bassetti, M.; Bellelli, V.; Bianchi, L.; Marincola Cattaneo, F.; Mazzocchetti, S.; Paciacconi, E.; Cottini, F.; Schiattarella, A.; Tufaro, G.; et al. Efficacy of a Fosfomycin-Containing Regimen for Treatment of Severe Pneumonia Caused by Multidrug-Resistant Acinetobacter Baumannii: A Prospective, Observational Study. Infect. Dis. Ther. 2021, 10, 187-200. [CrossRef]

96. Thabit, A.K.; Hobbs, A.L.V.; Guzman, O.E.; Shea, K.M. The Pharmacodynamics of Prolonged Infusion $\beta$-Lactams for the Treatment of Pseudomonas aeruginosa Infections: A Systematic Review. Clin. Ther. 2019, 41, 2397-2415. [CrossRef]

97. Gatti, M.; Pea, F. Pharmacokinetic/Pharmacodynamic Target Attainment in Critically Ill Renal Patients on Antimicrobial Usage: Focus on Novel Beta-Lactams and Beta Lactams/Beta-Lactamase Inhibitors. Expert Rev. Clin. Pharmacol. 2021, 14, 583-599. [CrossRef]

98. Sumi, C.D.; Heffernan, A.J.; Lipman, J.; Roberts, J.A.; Sime, F.B. What Antibiotic Exposures Are Required to Suppress the Emergence of Resistance for Gram-Negative Bacteria? A Systematic Review. Clin. Pharm. 2019, 58, 1407-1443. [CrossRef] [PubMed]

99. Pea, F.; Viale, P. Bench-to-Bedside Review: Appropriate Antibiotic Therapy in Severe Sepsis and Septic Shock-Does the Dose Matter? Crit. Care 2009, 13, 214. [CrossRef]

100. Gatti, M.; Pea, F. Antimicrobial Dose Reduction in Continuous Renal Replacement Therapy: Myth or Real Need? A Practical Approach for Guiding Dose Optimization of Novel Antibiotics. Clin. Pharm. 2021, 60, 1271-1289. [CrossRef] [PubMed]

101. Abdul-Aziz, M.H.; Alffenaar, J.-W.C.; Bassetti, M.; Bracht, H.; Dimopoulos, G.; Marriott, D.; Neely, M.N.; Paiva, J.-A.; Pea, F.; Sjovall, F.; et al. Antimicrobial Therapeutic Drug Monitoring in Critically Ill Adult Patients: A Position Paper. Intensive Care Med. 2020, 46, 1127-1153. [CrossRef] [PubMed]

102. Jager, N.G.L.; van Hest, R.M.; Lipman, J.; Taccone, F.S.; Roberts, J.A. Therapeutic Drug Monitoring of Anti-Infective Agents in Critically Ill Patients. Expert Rev. Clin. Pharmacol. 2016, 9, 961-979. [CrossRef] [PubMed]

103. Williams, P.; Cotta, M.O.; Roberts, J.A. Pharmacokinetics/Pharmacodynamics of $\beta$-Lactams and Therapeutic Drug Monitoring: From Theory to Practical Issues in the Intensive Care Unit. Semin. Respir. Crit. Care Med. 2019, 40, 476-487. [CrossRef] [PubMed]

104. Sandaradura, I.; Alffenaar, J.-W.; Cotta, M.O.; Daveson, K.; Day, R.O.; Van Hal, S.; Lau, C.; Marriott, D.J.E.; Penm, J.; Roberts, J.A.; et al. Emerging Therapeutic Drug Monitoring of Anti-Infective Agents in Australian Hospitals: Availability, Performance and Barriers to Implementation. Br. J. Clin. Pharmacol. 2021; Early View. [CrossRef]

105. Segala, F.V.; Bavaro, D.F.; Di Gennaro, F.; Salvati, F.; Marotta, C.; Saracino, A.; Murri, R.; Fantoni, M. Impact of SARS-CoV-2 Epidemic on Antimicrobial Resistance: A Literature Review. Viruses 2021, 13, 2110. [CrossRef] [PubMed]

106. Di Gennaro, F.; Marotta, C.; Amicone, M.; Bavaro, D.F.; Bernaudo, F.; Frisicale, E.M.; Kurotschka, P.K.; Mazzari, A.; Veronese, N.; Murri, R.; et al. Italian Young Doctors' Knowledge, Attitudes and Practices on Antibiotic Use and Resistance: A National Cross-Sectional Survey. J. Glob. Antimicrob. Resist. 2020, 23, 167-173. [CrossRef]

107. Tamma, P.D.; Aitken, S.L.; Bonomo, R.A.; Mathers, A.J.; van Duin, D.; Clancy, C.J. Infectious Diseases Society of America Guidance on the Treatment of Extended-Spectrum $\beta$-Lactamase Producing Enterobacterales (ESBL-E), Carbapenem-Resistant Enterobacterales (CRE), and Pseudomonas Aeruginosa with Difficult-to-Treat Resistance (DTR-P. Aeruginosa). Clin. Infect. Dis. 2021, 72, 1109-1116. [CrossRef]

108. Hawkey, P.M.; Warren, R.E.; Livermore, D.M.; McNulty, C.A.M.; Enoch, D.A.; Otter, J.A.; Wilson, A.P.R. Treatment of Infections Caused by Multidrug-Resistant Gram-Negative Bacteria: Report of the British Society for Antimicrobial Chemotherapy/Healthcare Infection Society/British Infection Association Joint Working Party. J. Antimicrob. Chemother. 2018, 73, iii2-iii78. [CrossRef]

109. Pouch, S.M.; Patel, G. AST Infectious Diseases Community of Practice Multidrug-Resistant Gram-Negative Bacterial Infections in Solid Organ Transplant Recipients-Guidelines from the American Society of Transplantation Infectious Diseases Community of Practice. Clin. Transplant. 2019, 33, e13594. [CrossRef] 
110. Peña-López, Y.; Ramirez-Estrada, S.; Eshwara, V.K.; Rello, J. Limiting Ventilator-Associated Complications in ICU Intubated Subjects: Strategies to Prevent Ventilator-Associated Events and Improve Outcomes. Expert Rev. Respir. Med. 2018, 12, 1037-1050. [CrossRef]

111. Magiorakos, A.-P.; Srinivasan, A.; Carey, R.B.; Carmeli, Y.; Falagas, M.E.; Giske, C.G.; Harbarth, S.; Hindler, J.F.; Kahlmeter, G.; Olsson-Liljequist, B.; et al. Multidrug-Resistant, Extensively Drug-Resistant and Pandrug-Resistant Bacteria: An International Expert Proposal for Interim Standard Definitions for Acquired Resistance. Clin. Microbiol. Infect. 2012, 18, 268-281. [CrossRef] [PubMed]

112. Murad, M.H.; Asi, N.; Alsawas, M.; Alahdab, F. New Evidence Pyramid. Evid. Based Med. 2016, 21, 125-127. [CrossRef] [PubMed] 